\title{
A systematic classification of Plasmodium falciparum P-loop NTPases: structural and functional correlation Deepti Gangwar ${ }^{\dagger 1}$, Mridul K Kalita ${ }^{\dagger 2}$, Dinesh Gupta ${ }^{2}$, Virander S Chauhan ${ }^{1}$ and Asif Mohmmed*1
}

Address: ${ }^{1}$ Malaria Group, International Center for Genetic Engineering and Biotechnology, Aruna Asaf Ali Marg, New Delhi-110067, India and ${ }^{2}$ Structure \& Computational Biology Group, International Center for Genetic Engineering and Biotechnology, Aruna Asaf Ali Marg, New Delhi110067, India

Email: Deepti Gangwar - deepti@icgeb.res.in; Mridul K Kalita - mridulkk@icgeb.res.in; Dinesh Gupta - dinesh@icgeb.res.in; Virander S Chauhan - virander@icgeb.res.in; Asif Mohmmed* - amohd@icgeb.res.in

* Corresponding author †Equal contributors

Published: 18 April 2009

Malaria Journal 2009, 8:69 doi:10.1 186/1475-2875-8-69

This article is available from: http://www.malariajournal.com/content/8/I/69

(c) 2009 Gangwar et al; licensee BioMed Central Ltd.

This is an Open Access article distributed under the terms of the Creative Commons Attribution License (http://creativecommons.org/licenses/by/2.0), which permits unrestricted use, distribution, and reproduction in any medium, provided the original work is properly cited.

\begin{abstract}
Background: The P-loop NTPases constitute one of the largest groups of globular protein domains that play highly diverse functional roles in most of the organisms. Even with the availability of nearly 300 different Hidden Markov Models representing the P-loop NTPase superfamily, not many P-loop NTPases are known in Plasmodium falciparum. A number of characteristic attributes of the genome have resulted into the lack of knowledge about this functionally diverse, but important class of proteins.
\end{abstract}

Method: In the study, protein sequences with characteristic motifs of NTPase domain (Walker A and Walker B) are computationally extracted from the $P$. falciparum database. A detailed secondary structure analysis, functional classification, phylogenetic and orthology studies of the NTPase domain of repertoire of 97 P. falciparum P-loop NTPases is carried out.

Results: Based upon distinct sequence features and secondary structure profile of the P-loop domain of obtained sequences, a cladistic classification is also conceded: nucleotide kinases and GTPases, ABC and SMC family, SFI/2 helicases, AAA+ and AAA protein families. Attempts are made to identify any ortholog(s) for each of these proteins in other Plasmodium sp. as well as its vertebrate host, Homo sapiens. A number of $P$. falciparum P-loop NTPases that have no homologue in the host, as well as those annotated as hypothetical proteins and lack any characteristic functional domain are identified.

Conclusion: The study suggests a strong correlation between sequence and secondary structure profile of P-loop domains and functional roles of these proteins and thus provides an opportunity to speculate the role of many hypothetical proteins. The study provides a methodical framework for the characterization of biologically diverse NTPases in the $P$. falciparum genome.

The efforts made in the analysis are first of its kind; and the results augment to explore the functional role of many of these proteins from the parasite that could provide leads to identify novel drug targets against malaria. 


\section{Background}

Despite encouraging advances in vaccine development, malaria remains the most serious and widespread parasitic disease of humans. Each year, approximately 300500 million people become infected with malaria and two to three million die as a result [1]. The availability of the complete genome sequence of Plasmodium falciparum, the causative agent of fatal cerebral malaria, has opened new avenues to identify genes important for the parasite's survival. This information can be utilized for the development of effective drugs or vaccines against the parasite. Unfortunately, nearly $60 \%$ of the $P$. falciparum genome (5411 proteins) has been designated as hypothetical proteins as they lack sequence similarity to any protein known to date [2]. This large and unexplored group of hypothetical proteins may contain proteins that play an important role in physiological pathways specific to the malaria parasite. The functional interruption of such proteins/pathways without deleterious consequences to the host should be the one of the primary tasks in data mining. A pipeline of systematic studies is thus required to elucidate the functional relevance of such proteins in the parasite's survival.

A number of these hypothetical proteins (unknown protein function) in P. falciparum genome contain a P-loop NTPase domain. Briefly, the P-loop NTPases constitute a large super-family of proteins [3] and are involved in disparate physiological processes. For example, processes include translation, transcription, replication and DNA repair, intracellular trafficking, membrane transport and activation of various metabolites [3-5]. The P-loop NTPases carry out such diverse cellular functions by hydrolyzing the $\alpha-\beta$ phosphate bond of a bound nucleotide triphosphate i.e. ATP or GTP [6].

Based on amino acid sequence, the P-loop NTPase fold is characterized by the presence of a $\mathrm{N}$-terminal Walker A motif, represented by a flexible loop joining a $\beta$-strand and an $\alpha$-helix. The loop typically adopts the sequence pattern GxxGxGK [ST], whose function is to properly position the triphosphate moiety of a bound nucleotide. The distal Walker B motif (hhhhDE) contains a conserved aspartate (less commonly glutamate residue). The motif is situated terminally in a $\beta$-strand and binds a waterbridged $\mathrm{Mg}^{2+}$ ion [5,7]. On structural aspects, the P-loop NTPases are $\alpha-\beta$ proteins that contain regularly recurring $\alpha-\beta$ units with the five $\beta$-strands $(\beta 1-\beta 5)$. The $\beta$-strands forms a central core arranged in the order $\beta$ (5-1-4-3-2) or $\beta$ (5-1-3-4-2), surrounded by $\alpha$-helices on both sides [8,9]. The P-loop NTPases can be divided into two groups: one group includes the nucleotide kinases and the GTPases where the $\beta$-strand leading to the P-loop and the Walker B strand are direct neighbors. The second group includes AAA, ABC, SF1/2 helicases and RecA/F1 ATPases and is characterized by an additional $\beta$-strand inserted between the P-loop strand and the Walker $B$ strand [10-12]. Despite these basic common sequence and structural features, the P-loop NTPases exhibit extreme sequence divergence. The huge sequence diversity had so far hampered a clear understanding of the phylogenetic relationships within these P-loop proteins. The entire complement of the P-loop NTPases of an organism varies considerably in sequence and the functional aspects. Therefore, identification and phylogenetic classification of the P-loop NTPases of an organism may provide insights into distinct physiological processes involving these proteins. Since the malaria parasite resides inside the host cells during most part of its life cycle, some of these processes such as trafficking of proteins and translocation of metabolites might be unique to the parasite and involve P-loop NTPases. In the present study, a comprehensive survey of the P-loop NTPases in $P$. falciparum is carried out by analyzing the sequence and structural features of the NTPase domain of functionally diverse proteins. Further, based on these identified features, a few of these hypothetical proteins are classified. For robustness of the analysis, the traditional cladistic and phylogenetic tree approaches (based on sequence and structural motifs of P-loop NTPases) are also combined. The study underlines the evolutionary information in addition to other sequence-structure features and thus, to develop a systematic classification of the P-loop NTPases in the P. falciparum. The study has also facilitated identification of some of the parasite specific $P$ loop-NTPases as putative novel drug targets against the parasite.

\section{Methods \\ Retrieval of sequences with classical Walker $A$ and Walker B motifs}

An initial computational search of the complete P. falciparum genome for the Superfamily: P-loop NTPase (SSF52540) revealed 302 proteins; 67 out of which are annotated as hypothetical proteins. In addition, to extract the proteins with classical Walker A and Walker B motifs and their variants, the motif search tool of PlasmoDB release $4.4 \mathrm{http}: / / \mathrm{v} 4-4$.plasmodb.org/restricted/plasmod bmotif.shtml is used. These two motifs are represented by GxxGxGK [TS] and hhhhDE patterns, respectively where ' $x$ ' is any of the 20 amino acids and ' $h$ ' is any hydrophobic residue. It led to the inclusion of those proteins which have been annotated as P-loop NTPases by PlasmoDB and Pfam databases. From this analysis, an initial set of $120 \mathrm{P}-$ loop NTPase sequences containing both Walker motifs is obtained. Further, for each of these proteins; the sequence regions corresponding to the P-loop domain only (comprising both Walker regions) are extracted. Variation in the sequence length (171 to 8094 amino acids) and Walker A and Walker B motifs is observed for most of these proteins. The unavailability of crystal structures of 
most of these proteins is a major hindrance in the structure based multiple alignments and their systematic analysis; hence only 97 proteins in which the P-loop domain encompasses a length of 300 to 400 residues are studied, to facilitate in sequence based multiple alignments (Additional file 1). The refined dataset is then used for secondary structure and phylogenetic analyses, followed by classification and orthology studies. Apart from above analysis, the protein sequences are also screened using signalP 3.0, TMHMM v2.0 and TMpred tools to predict signal and transmembrane domains. The absolute expression profiles of these proteins that are temperature synchronized are also obtained from PlasmoDB.

\section{Functional classification using Pfam v2 I.0}

Since the P-loop NTPases are known to belong to functionally diverse groups therefore a domain analysis of dataset of 97 P. falciparum P-loop proteins is performed for functional classification of these proteins using Pfam v21.0 database of domains. The proteins are functionally correlated based on the domains present with E-values with a threshold of $10^{-2}$.

\section{Secondary structure analysis of proteins}

To perform secondary structure analysis of the P-loop domain of the dataset of 97 proteins, a representative dataset of 533 seed protein sequences is generated pertaining to Pfam accession ID: PF00004, PF07724, PF07726, PF04326 and PF07728. The secondary structure is predicted by the standalone version of PSIPRED v2.0, which is a simple and reliable method, incorporating two feed-forward neural networks that perform an analysis on output obtained from the initial run of PSI-BLAST at the cut-off E-value of 0.001 (Blast v2.2.4) [13]. PSIPRED predicts the secondary structure for each residue and provides a confidence score for three types of secondary structures: helix, sheet and coil. The sequences are aligned according to the secondary structure for AAA+ proteins to classify them into classical AAA or AAA+ and other super-families based on the structural conformations and domain organizations. Multiple alignments are obtained using CLUSTALW (1.83) [14-16], which are manually refined to reflect the available structural information.

\section{Phylogenetic analysis}

The phylogenetic relationships amongst various classes of proteins are determined using the Phylip 3.67 package [17]. PROTDIST is used on the 97 sequences to calculate a distance matrix according to the Dayhoff PAM probability model [18]. The computed distances represent the expected fraction of amino acid substitutions between each pair of sequences. The distance matrix is then used to estimate phylogenies using the neighbour joining (NJ) method. Bootstrapping is carried out using SEQBOOT $(1,000$ replicates for the PAM model of substitution). CONSENSE is used to compute the consensus tree by the majority rule method. The final unrooted tree diagram is generated using TreeView http://taxonomy.zool ogy.gla.ac.uk/rod/treeview.html.

\section{Identification of orthologous protein sequences using OrthoMCL}

To identify the possible drug targets in terms of orthology of proteins between $P$. falciparum and its human host and with other Apicomplexans, OrthoMCL algorithm is used [19], which performs the Markov Clustering (MCL) to group orthologs and paralogs across proteins of multiple organisms. The stand-alone version 1.3 of OrthoMCL is obtained from http://www.orthomcl.org/cgi-bin/ OrthoMclWeb.cgi. Protein FASTA files of each of the genome is given as input to the algorithm. An all-againstall BLASTP analysis (at E value of $10^{-5}$ ) is carried out using OrthoMCL. The blast output, which describes genes paired by BLAST matches, the E-value, and the identity percentage and the related HSP information, is parsed to OrthoMCL. The evolutionary related proteins are interlinked in a similarity graph matrix. MCL http:// micans.org/mcl/ is then invoked to split mega-clusters as an analogous process of manual review in COG construction (Clusters of Orthologous Groups). As a result, different clusters of orthologous proteins are created.

\section{Results and discussion \\ Sequences with P-loop motifs: Generation of dataset}

Using consensus sequences of Walker A (GxxGxGK [ST]), Walker B (hhhhDE) motifs and their variants along with other proteins annotated as P-loop NTPases (PlasmoDB), a dataset of $97 \mathrm{P}$. falciparum protein sequences is generated. These proteins showed variations in the length of the P-loop NTPase domains having insertions and repeat regions between Walker $A$ and Walker $B$ motifs. In addition, some of the proteins showed variations in the P-loop motifs compared to canonical forms, suggesting their divergence from other P-loop NTPases. These sequences are further analysed for protein domain organization, secondary structure and phylogenetic and orthologous relationships. A list of all the 97 proteins and their characteristics is provided in Additional file 2.

\section{Functional classification based on domains and secondary structural analysis}

Analysis of the secondary structures of the P-loop NTPase domain and presence of the Pfam domains served as the basis for functional classification of these proteins. Typically, the functional groups have been defined based upon the presence of Pfam domains and the hierarchical classification of individual proteins is carried out on the basis of structural analysis of P-loop domains in these proteins using PSIPRED (Figure 1) [20,21]. Based upon these studies; the repertoire of P. falciparum P-loop NTPases is classified into clades, families and subfamilies (Additional file 2). 


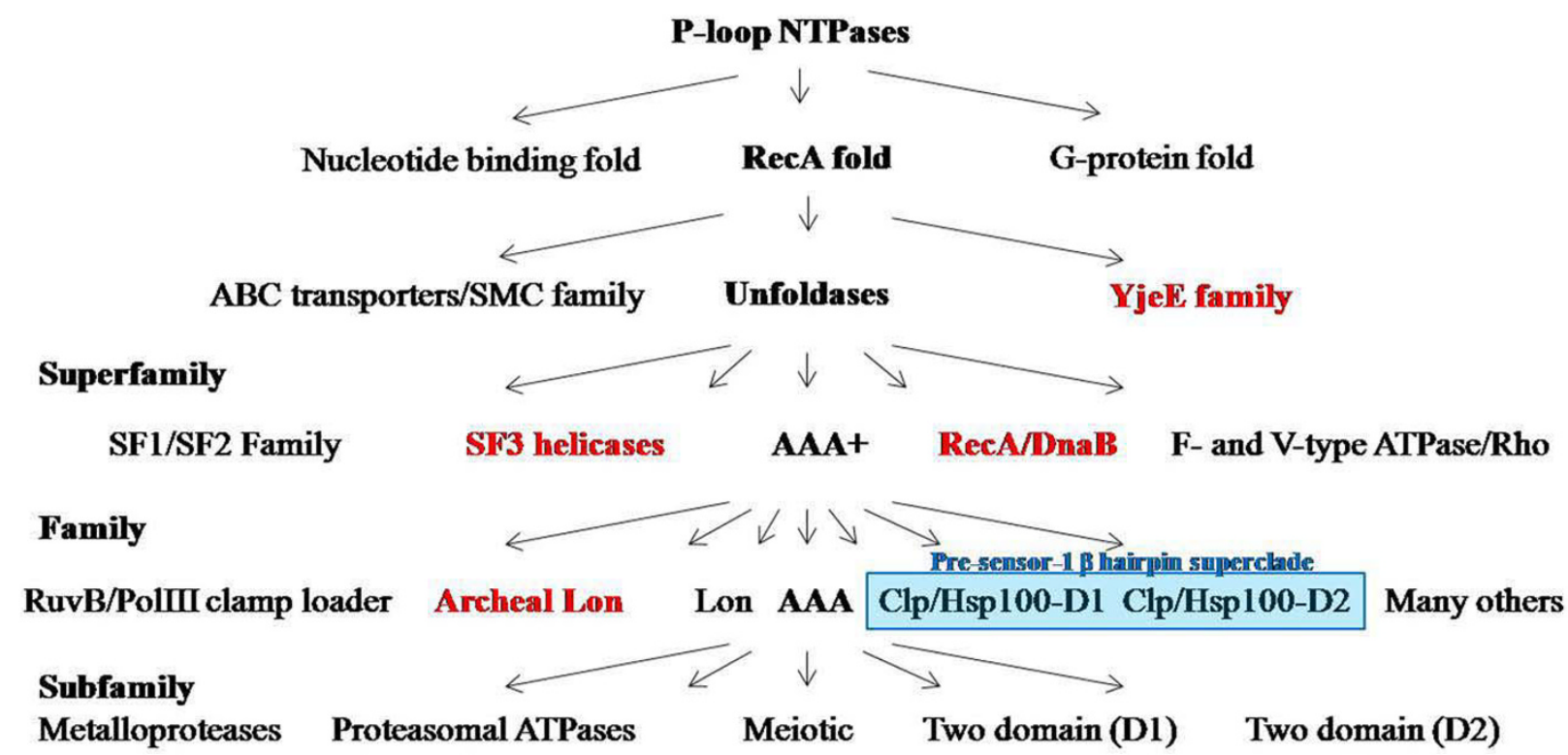

Figure I

Schematic representation of classification of P-loop NTPases into families, super families and clades (following Leipe et al., 2002; 2003). The families marked in red are not observed in Plasmodium falciparum. The clades that together form the Pre-sensor-I $\beta$ hairpin superclade are highlighted in blue.

\section{Kinase GTPases}

In this class two myosin binding proteins of molecular motors, PFL1435c (myosin d) and PF13_0233 (myosin a) are identified. Unlike other myosins, PFL1435c contains long asparagine repeats between the Walker A and Walker B motifs. The orthologs of PF13_0233 protein are also found to be present in other Plasmodium species as well as in a closely related apicomplexan organism (Toxoplasma gondii); suggesting its evolutionary conservation. Four other kinases are also identified (MAL13P1.148, PF11_0416, PFE0175C and PFF0675c), that showed major variation in the P-loop domain organization; the Ploop domain of these proteins lack a classical Walker B motif. Although a total of 99 protein kinases have been identified in P. falciparum genome [22], only PF13_0334 is found to contain the classical P-loop NTPase fold.

The P. falciparum GTPases that have been classified within P-loop NTPases include PF11_0183, a nuclear binding protein and two transcription initiation and elongation factors, PFA0595c and PF13_0069. Based upon Pfam domain analysis along with their secondary structures, the two hypothetical proteins (PFF0810c and PF14_0052) are also classified as Kinase GTPases.

\section{ASCE/RecA fold division}

\section{$A B C$ transporters and SMC family}

ABC transporters are part of a large ATP binding protein family that plays physiological function of translocation of metabolites across the membranes. In P. falciparum, 15 $\mathrm{ABC}$ transporters are identified that contain core $\beta$-strands and helices along with sensor-1 residue, which intercalates between the P-loop and aspartate residue of the Walker B motif. The characteristic signature 'LSGG' of ABC proteins, located upstream of the Walker B motif is found in only five $P$. falciparum ATPases while a hydrophobic residue substitution primarily at the first position of the motif is noticed in a majority of remaining proteins (Figure 2). Based upon these sequence features and secondary structure analysis these $15 \mathrm{ABC}$ transporters are phylogeneticaly assigned into subfamilies following [23]. Out of these $15 \mathrm{ABC}$ proteins, three are full transporter [with two transmembrane domain (TMD) and two nucleotide binding domain (NBD)] PFC0875w, PFE1150w and PF14_0455; and eight are identified as half transporters (with one TMD and one NBD) PF13_0218, PF13_0271, PFL0495c, PFA0590w, PF11_0466, PFL1410c, PF14_0133 and PF14_0244; whereas four other ABC proteins are found to lack the transmembrane domain. Based on these observations, PF13_0218, PF13_0271, PFL0495c, PF14_0455 and PFE1150w have been assigned to the ABCB subfamily, which is known to include multidrug resistant proteins (MDR/TAP). These P-glycoprotein (PgP) homologues of the P. falciparum are found to be similar to the mammalian MDR proteins and have been implicated in providing parasite resistance against antimetabolites [24]. ABC transporters have also been implicated to export proteins (responsible for host modifica- 


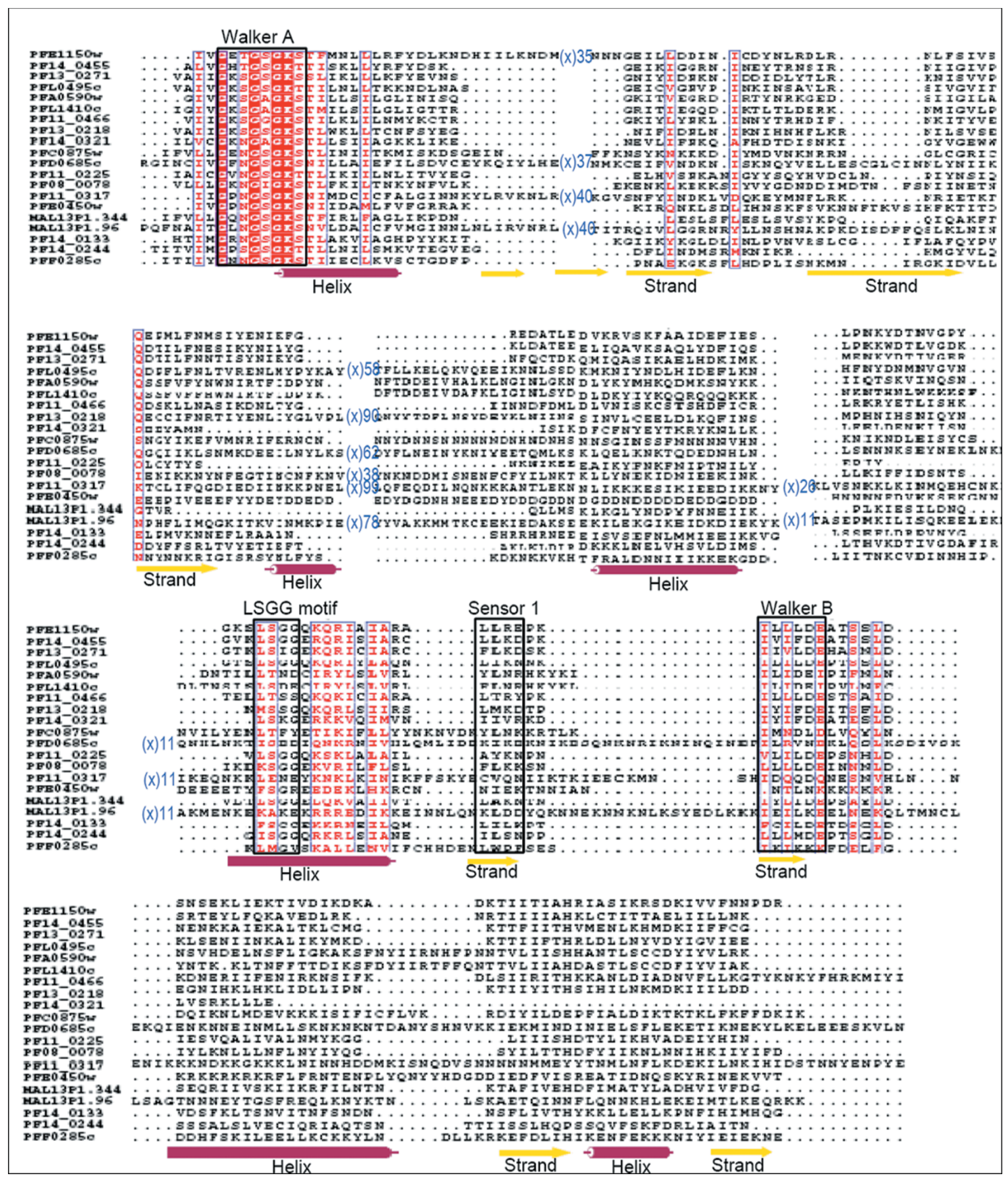

Figure 2

Multiple sequence alignment of P-loop NTPase domain of $P$. falciparum ABC transporters and SMC proteins. Conserved residues are in solid red and conserved motifs are boxed in black. The corresponding secondary structure is shown below the alignment. Purple cylinder represents helices and yellow arrows represent $\beta$-strands. 
tion, virulence and immune responses) in the host via alternative pathways [25]. The PF11_0466 protein belonging to $\mathrm{ABCB}$ subfamily, is an $\mathrm{ABC}$ transporter, expressed in late trophozoite stages [26] and contains a hydrophobic $\mathrm{N}$-terminal signal sequence. The $\mathrm{N}$-terminal signal sequence is known to be responsible for default trafficking of a parasite protein through endoplasmic reticulum-Golgi apparatus pathway to the parasitophorous vacuole in absence of any organelle targeting signals. It is not clear if PF11_0466 protein is localized to mitochondria or apicoplast; in absence of targeting to any of these organelles it may localize in the plasma membrane or parasitophorous vacuolar membrane and might have novel function(s) in protein trafficking. Two other $P$. falciparum ABC tarns porters, PFA0590w and PFL1410c are suggested to be involved in redox metabolism and might have functional activities of MRP (MDR associated protein) and/or GSSG pumps [27]. These characteristics suggested that these proteins may be classified under the ABCC subfamily. The mammalian MRPs belonging to the ABCC family are associated with extrusion of oxidized glutathione (GSSG) generated during glutathione synthesis, a major element of antioxidant defense for the parasite [28]. Interestingly, the protein PFL1410c showed no homology with any of the known human MRPs and hence can be studied as a potential drug target. PF14_0244 is assigned to the ABCG subfamily a reverse transporter with N-terminal NBD and C-terminal TMD. Further, the proteins without transmembrane region have been classified under the ABCF subfamily; members of this family are not known to be involved in any membrane transport functions [23].

Along with the $\mathrm{ABC}$ transporters, SMC (Structural Maintenance of Chromosomes) proteins also represent a large family of ATPases involved in chromosome organization like the sister-chromatid cohesion and DNA repair [27]. Apart from high sequence similarity, SMCs also share structural similarity with $\mathrm{ABC}$ proteins (Figure 3 ). Five $P$. falciparum proteins are annotated as putative SMC proteins (PlasmoDB). The P. falciparum SMC proteins are found to be highly conserved amongst Plasmodium species which suggests that they may play an important role as a component of chromosomal maintenance complex and are probably indispensable for the parasite. Although $\mathrm{ABC}$ transporter proteins are functionally different from the SMC proteins, Figure 2 clearly illustrates that the $\mathrm{N}$-terminal region of proteins belonging to these two groups is significantly conserved near the Walker-A motif, extended by two small conserved regions having charged residues (Lys, Arg, Glu) flanked by hydrophobic (Leu, Ile, Ala) or polar residues (Ser, Asn, Gln). Based on secondary structure analysis, three SMC proteins (PFD0685c, MAL13P1.96 and PF11_0317) clustered together as compared to PFF0285c; probably due to two long stretches of continu- ous helical regions in the PFF0285c protein. Evolutionary studies that the ATP binding domain of proteins belonging to the same $\mathrm{ABC}$ subfamily are more closely related to each other than to those belonging to other subfamilies, suggesting their concerted evolution whereas SMC proteins clustered together away from $\mathrm{ABC}$ families and have evolved further from these subfamilies (Figure 3 ).

\section{Unfoldases}

The unfoldase division of ASCE/RecA fold proteins is characterized by the core P-loop containing Walker A and $\mathrm{B}$ motifs, and the presence of arginine finger at the $\mathrm{N}$-terminus of $\beta 5$ (Figure 4 ). The division includes SF1/SF2 helicases, F- and V-type ATPases/Rho and AAA+ superfamily of proteins.

\section{SFI/SF2 Helicases}

Helicases are enzymes that unwind duplex DNA or RNA coupled to nucleoside 5' triphosphate (NTP) binding and hydrolysis [29]. Structural and sequence comparison of these proteins from different organisms have identified seven to nine short, conserved motifs called helicase signature motifs [30]. Most of the known 3'-5' DNA helicases are members of SF1 or SF2. These two superfamilies have similar sets of conserved motifs that are responsible for coupling of ATP hydrolysis to DNA translocation and unwinding. However, sequence homology across SF1 and SF2 families is very weak and limited to the signature sequences, required for NTP binding (Walker A and B motifs) [31]. ATP hydrolysis by SF1 helicases is stimulated only by ssDNA, whereas the ATPase activity of SF2 helicases is stimulated by both ssDNA and dsDNA [32]. In the present study, seven P-loop NTPases in the P. falciparum genome are found to contain helicase, HA2 and DEAD/ DEAH domain(s), characteristic of DNA/RNA helicases. The secondary structures of these helicases are dominated by helices towards the $\mathrm{N}$-terminal, followed by regions having alternate $\alpha$-helices and $\beta$-sheets. Multiple sequence alignments revealed that although the DEAH box helicases had high sequence conservation throughout the P-loop domain, PF08_0042 and PFC0440c had an 'I' $\rightarrow$ ' $\mathrm{A}$ ' replacement in the DEAH box (Figure 4). In addition to some hydrophobic residue conservations among sequences, a sequence pattern 'I [LI]DE [AVIL] is found to be highly conserved. Downstream to the observed pattern, a Ser/Thr residue is found to be well-conserved amongst all the P. falciparum helicases.

\section{AAA+ superfamily}

The proteins belonging to AAA+ superfamily are highly diverse and are involved in varied cellular functions [33], but have a common AAA (ATPases Associated with diverse cellular Activities) structural fold [9]. The AAA+ domain contains a characteristic region of high sequence conservation called as the second region of homology (SRH) that 


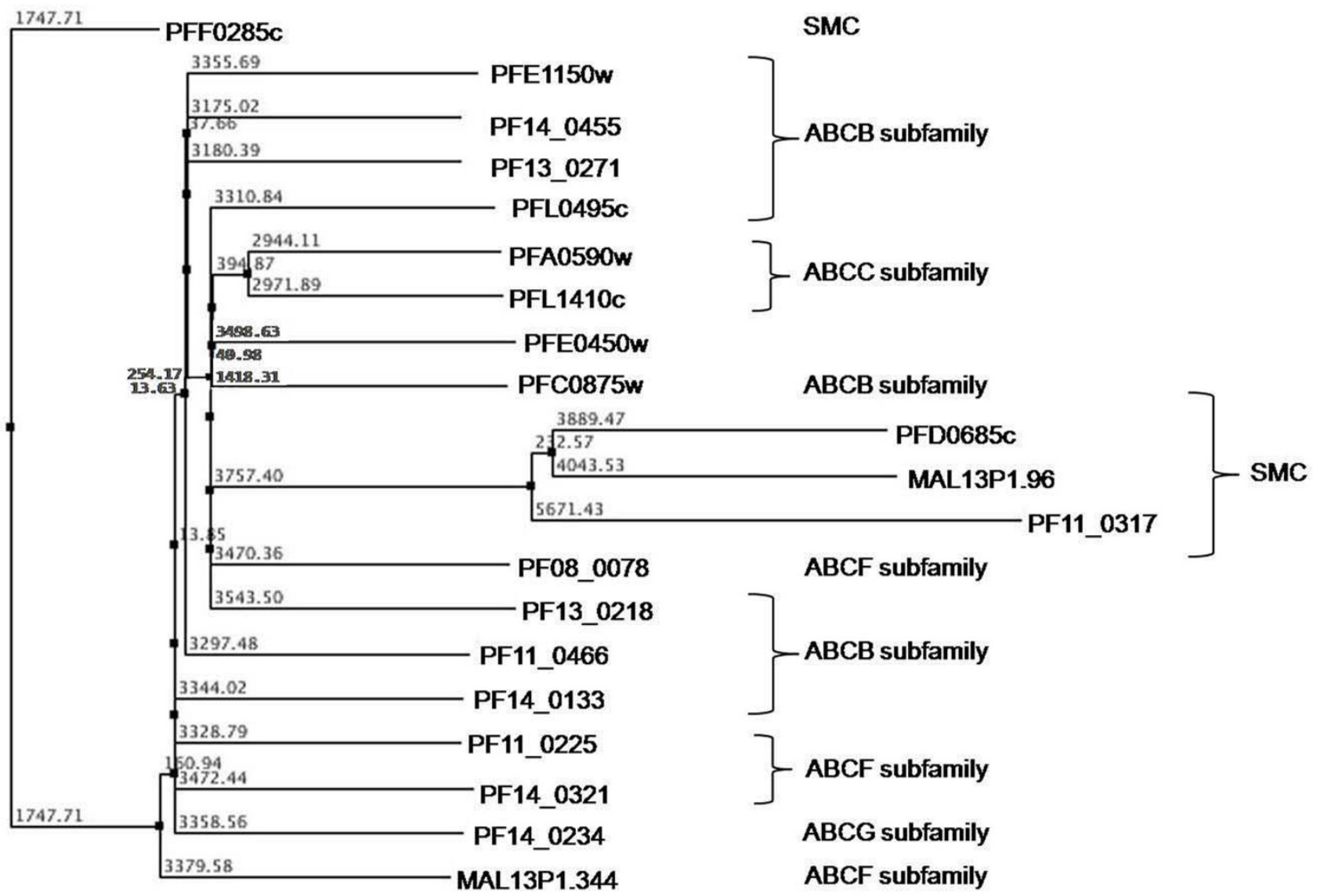

Figure 3

Phylogenetic classification of $P$. falciparum ABC transporters and SMC proteins based upon sequence and secondary structure homology among their P-loop NTPase domain. The branch numbers represents the distance values in $\mathrm{NJ}$ tree of protein sequences.

distinguishes AAA+ proteins from other P-loop NTPases. The SRH includes a polar residue at its N-terminal known as 'sensor 1' and an 'arginine finger' at the C-terminal helical domain that carries a conserved arginine residue known as 'sensor 2' [34] (Figure 5). The AAA+ superfamily include classical AAA proteins as well as proteins having divergent sequences but classical AAA structural fold. The $\mathrm{AAA}+$ superfamily has been divided into several different clades. The $P$. falciparum AAA+ proteins have been grouped based upon their sequence and structural features and discussed according to their respective functional clades.

\section{Clamp loader/RFC clade}

The clamp loader ATPases have a synapomorphic 'SRC' signature associated with the arginine finger [35]. In the preset study, five P. falciparum RFC (replication factor C) proteins are identified following the Walker A and B consensus analysis. Three of these proteins (PF14_0601,
PFL2005w and PFB0840w) share high sequence similarities as compared to other two (PFA0545c and PFB0895c). Further, the sequence analysis revealed that the sensor- 2 is conserved among all the five RFC proteins while the SRH is conserved in only three proteins (PF14_0601, PFL2005w and PFB0840w). The structural analysis revealed one hypothetical protein (PF14_0326) having secondary structure similar to that of known RFC proteins. This protein showed a single residue substitution in the Walker B motif; ' $W$ ' residue replacing A in the $\{$ AVIL\} pattern (Figure 4). In order to analyse the correlation between the secondary structure of the P-loop domain and functional role of a protein, the helicases and the RFC proteins are studied together. The sequence pattern 'I [LI]DE [AVIL], that is observed for helicases, is also found to be conserved in the RFC proteins. The evolutionary analysis of the helicases along with the replication factor $\mathrm{C}$ (RFC) proteins revealed that helicases proteins have a tendency to cluster. The only exception is MAL13P1.14 


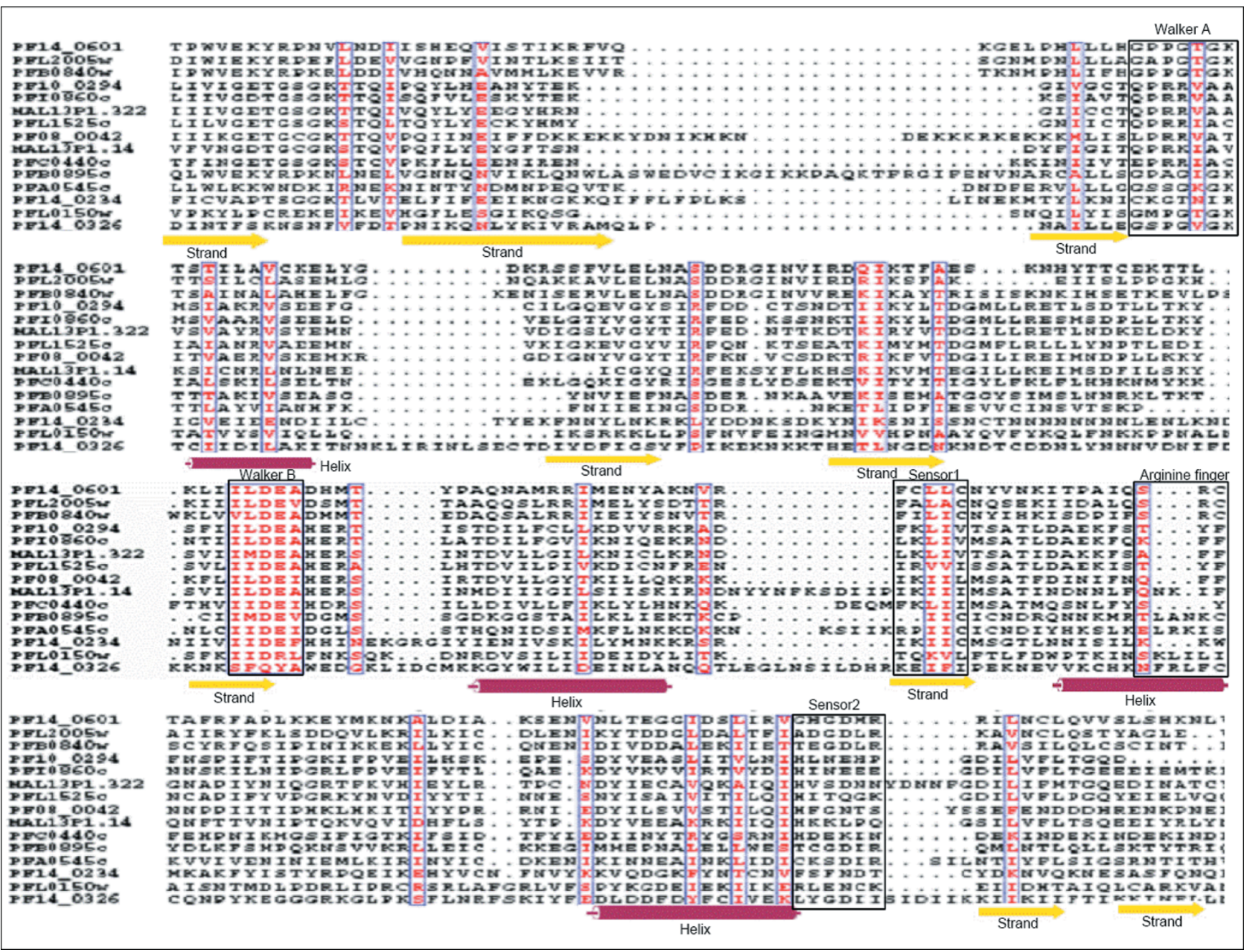

Figure 4

Multiple sequence alignment of P-loop NTPase domain of $P$. falciparum SF I/SF2 Helicases and Replication factor C (RFC) proteins. Conserved residues are in solid red and conserved motifs are boxed in black. The corresponding secondary structure is shown below the alignment. Purple cylinder represents helices and yellow arrows represent $\beta$-strands.

protein, which falls out of the cluster probably due to extended sequence length in the $\mathrm{N}$-terminal of its $\mathrm{P}$-loop NTPase domain. Three RFC proteins are observed to group closer with the helicases as compared to the other RFC proteins (Figure 6). These results indicate that helicases and RFC proteins might have undergone a convergent evolution since majority of the helicases are found to be similar both at the sequence as well as in the structural organization of the P-loop domain with the RFC proteins.

\section{DnaA/CDC6/ORC clade}

This clade is defined by the presence of two $\alpha$-helices after strand 2 (of approximately equal size) that are packed against each other [35]. The only representative of this family is PFL0150w. The N-terminus of the protein had rich low complexity regions and the ATPase domain had secondary structure similar to that of other AAA+ proteins (Figure 6).

\section{ClpA/B ATPase clade}

Members of the ClpA/B ATPases family contain two AAA+ domains that vary from each other in terms of sequence and phylogenetic affinities. The ClpB ATPases contain a middle domain ( $\mathrm{M}$ domain) between the two ATPase domains as compared to ClpA. The ClpB ATPases play role of important chaperones whereas ClpA ATPases interact with Clp proteases to carry out regulated protein degradation [36]. Two P. falciparum proteins (PF14_0063 and PF11_0175) have been annotated as ClpA/B ATPases by PlasmoDB. However, PF14_0063 is found to contain substitution in some of the functionally conserved residues including $\mathrm{R} \rightarrow \mathrm{E}$ substitution in the arginine finger and $\mathrm{R}$ 


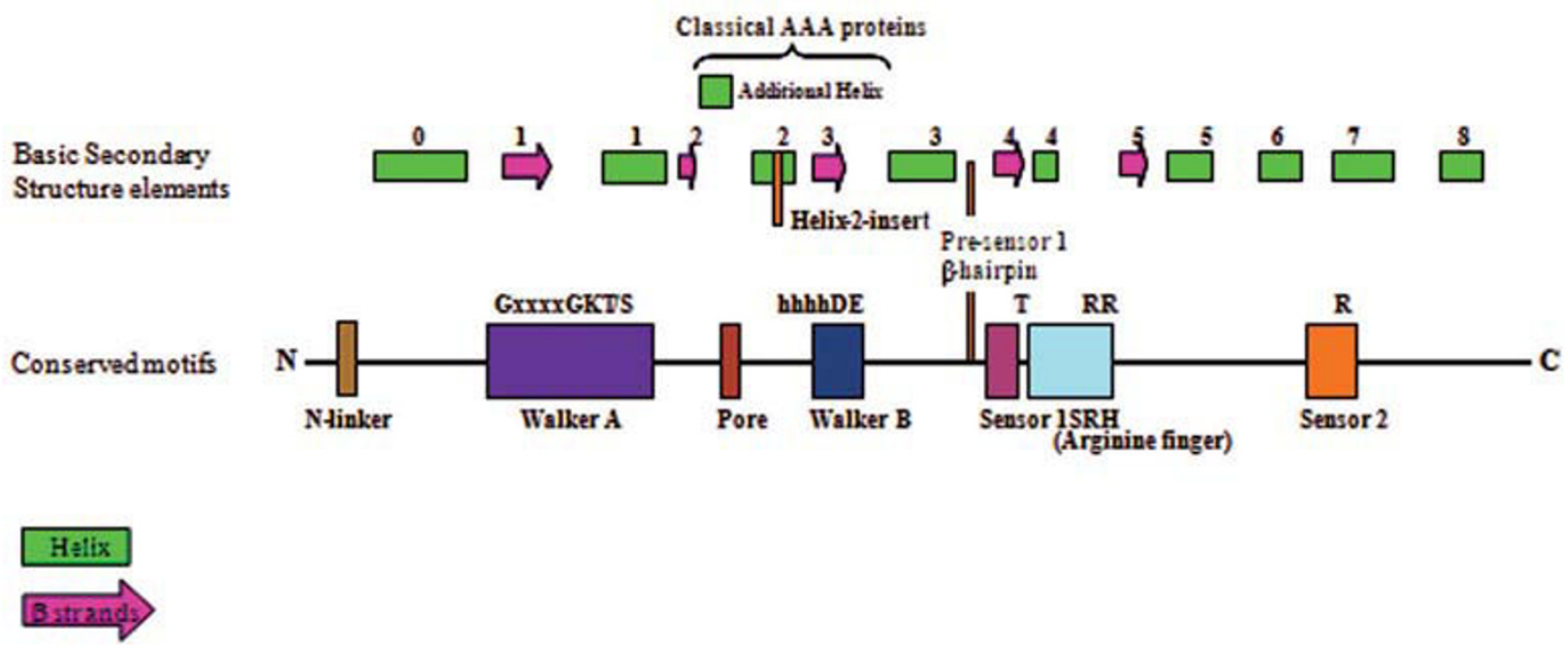

Figure 5

A schematic showing the organization of secondary structure and key elements of AAA+. Helical insertions and other variations observed in the domain organization are depicted. The location of characteristic residues within the motifs is marked.

$\rightarrow$ A substitution in the sensor 2 region which might render it non-functional. PF08_0063 is identified as another ClpA/B (annotated as hypothetical protein in the PlasmoDB) that also contains the $\mathrm{ClpN}$ domain and showed secondary structural organization and domain pattern as other ClpA/B proteins (Figure 7). Evolutionary studies nested these plasmodial ClpA/B homologs within the $\mathrm{ClpA} / \mathrm{B}$ proteases of bacterial lineage suggesting that they have been acquired from the prokaryotic precursors (Additional file 3). The AAA domain of the P. falciparum $\mathrm{ClpA} / \mathrm{B}$ proteins shared the characteristic features of the classical AAA clade in having the additional small helix downstream of strand 2 except the conserved glycine at the $\mathrm{N}$-terminal of arginine finger. The secondary structure of AAA module of parasite ClpA/B homologs clustered them separately from rest of the AAA proteins (Figure 8). Importantly, the absence of any human ClpA/B homologue renders the Plasmodium ClpA/B ATPases as important drug targets.

\section{"Pre-sensor-I $\beta$ hairpin" (PSIBH) superclade}

The remaining lineages of AAA+ namely HslU/ClpX/Lon, MCM, dynein/midasin and other relatives have been unified into a one large monophyletic group. The entire superclade is defined by the presence of an insert between the sensor-1 strand and the preceding helix known as "pre-sensor-1 $\beta$ hairpin" [35]. In this superclade, two distinct clades are identified, which are further divided into three protein families in the parasite genome.

\section{HsIU/CIpX/lon clade}

The HslU/ClpX clade proteins contain one ATPase domain as compared to ClpA (with two ATPase domains). The ATPase domain of HslU is interrupted by an 'I' domain involved in the substrate binding $[37,38]$. This clade is supported by an extended loop between strand-2 and helix-2. The HslU (ClpY) and ClpX ATPase interact with the protease partner HslV (ClpQ) and ClpA respectively to form multimeric protease degradation complex machineries as in case of ClpAP. Although ClpX ortholog is absent in P. falciparum, the ortholog of HslU (PFI0355c) annotated as ATP-dependent heat shock protein is identified. HslU orthologs are known in apicomplexans and kinetoplastids are predicted to be localized in the mitochondria or plastid [39]. Sequence and structural analysis of PfHsIU confirmed the presence of three characteristic domains of HslU proteins, N-terminal domain (N-domain), Intermediate domain (I domain) and C-terminal domain (C-domain) [40]. The HslU ortholog is an ATP binding regulatory subunit of prokaryotic proteasome complex with HslV/ClpQ threonine proteases. The ortholog of the prokaryotic HslV protease in the malaria parasite has been recently identified and is shown to be functionally important in the parasite [41]. Both Hslu and HslV lack any homolog in the vertebrate host and may be considered as a promising drug targets against the parasite. The Lon proteins from archea and bacteria define another family (LON family) within this clade. The PF14_0147 protein is identified as a $P$. falciparum Lon protease that contains the characteristic LAN domain and a Lon-protease domain flanking the AAA domain at the $\mathrm{N}$ and C-termini, respectively.

Helix-2 insert clade

As the name suggests, the defining feature of the clade is an insert in helix-2 that folds into two $\beta$-strands [35]. In 

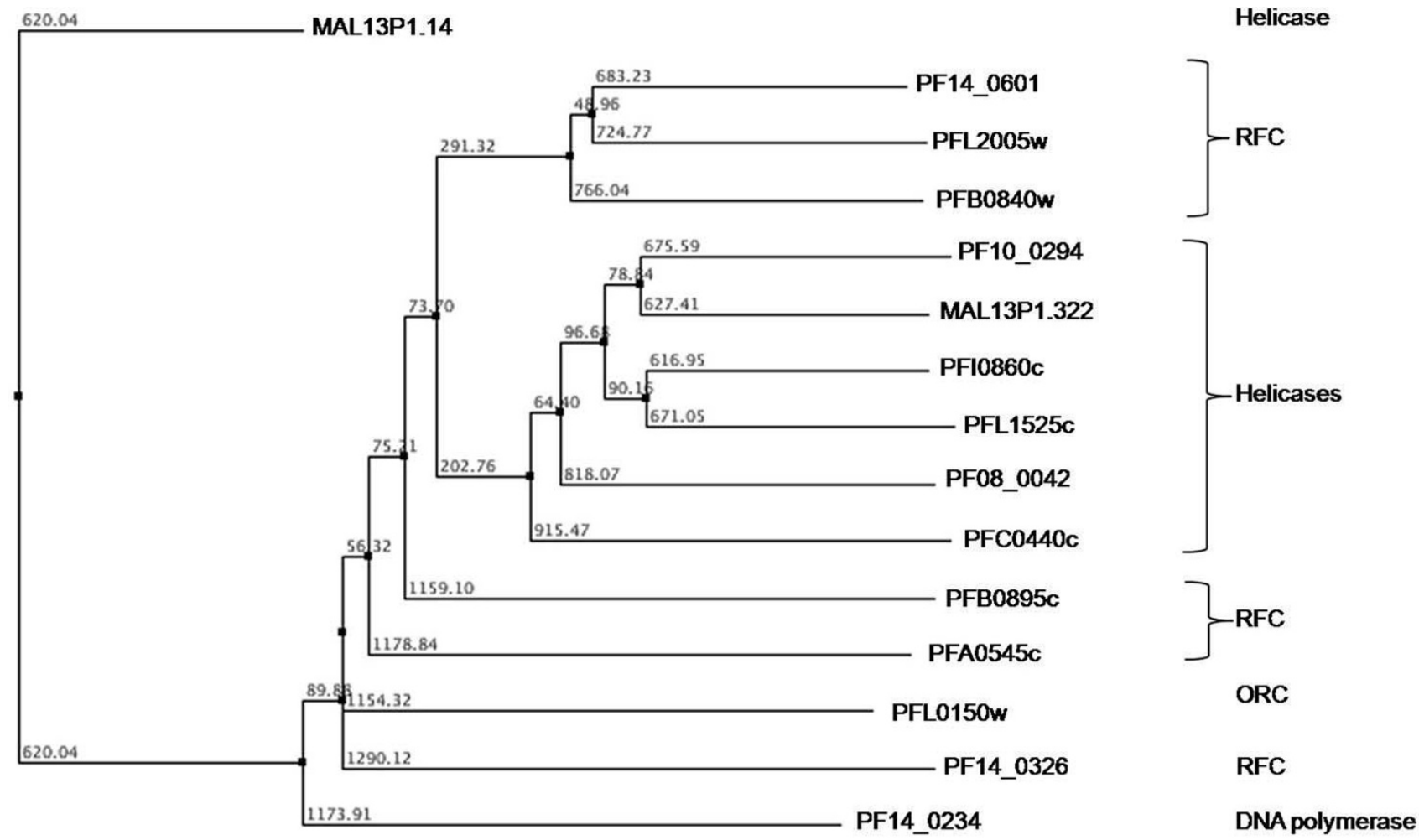

Figure 6

Phylogenetic classification of $P$. falciparum SF I/SF2 Helicases and Replication factor C (RFC) proteins based upon sequence and secondary structure homology among their P-loop NTPase domain. The branch numbers represents the distance values in $\mathrm{NJ}$ tree of protein sequences.

the $P$. falciparum genome, the helix-2 insert clade is represented by Mini Chromosomal Maintenance proteins (MCMs) and dyneins, whereas other members of the clade such as NtrC, YifB and MoxR are not found in the parasite genome.

\section{MCM family}

The MCM proteins are essential DNA replication initiation factors and the best characterized among them is a family of six structurally related proteins (MCM2-7) [42]. In the P. falciparum database three proteins (PF14_0177, PFD0790c and PFL0560c) have been annotated as MCMs belonging to the MCM2/3/5 family (PlasmoDB). Five other members of the MCM family (PF07_0023, PF13_0095, PF13_0291, PFE1345c and PFL0580w) that have certain minor deviations from the canonical form of P-loop motifs like Gxx [G/x]x [G/A]KS sequence of the Walker A motif are observed. The secondary structure analysis of the AAA region of all these MCM proteins revealed the classical helices and strand pattern. An invariably conserved sequence motif 'GLT' (for the observed pattern of ' $[\mathrm{AG}][\mathrm{FL}] \mathrm{T}$ ' in other organisms) in helix-2 region before the beta-alpha-beta insert is observed. The beta insert in the helix-2 of MCM family members of the malaria parasite is predominantly rich in hydrophobic residues except highly conserved charged residues of ' [KR]D' and 'LE', 5th and 14th positions, respectively, downstream of the GLT motif (Figure 9).

\section{Dynein family}

Dyneins are large molecular motor that transport various cellular cargo by "walking" along cytoskeletal microtubules towards the minus-end of the microtubule, which is usually oriented towards the cell center. Thus, they are called "minus-end directed motors". They contain six tandem AAA+ domains in the same polypeptide chain $[9,43]$. The parasite genome contains seven representatives (average length of $\sim 6000$ amino acids) from this family out of which only four proteins (PF14_0626, MAL7P1.162, PF10_0224 and PF11_0240) are annotated at PlasmoDB. Three other proteins PFI0260c, PFL0115w and MAL7P1.89 annotated as hypothetical proteins at PlasmoDB, are identified as dyneins based upon the protein domain analysis. These proteins have two dynein heavy 


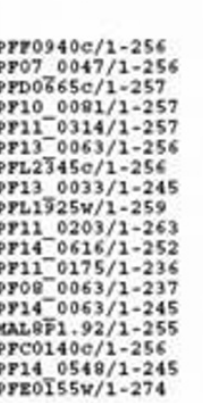

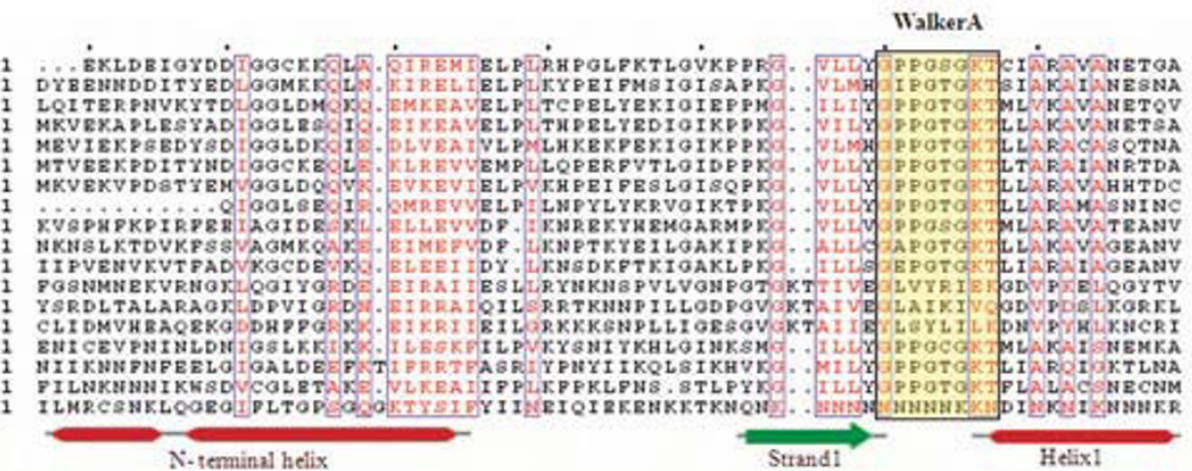

PYP0940e/1-256 PF $070047 / 1=256$ PFD $0365 \mathrm{C} / 1=257$ PF10-0081/1-257 $P Y 11^{-} 0314 / 1-257$
$P Y 13-0063 / 1-256$ PF13-0063/1-256 PFL 23450/1-256
PF13 $0033 / 1-245$ PF $13-0033 / 1-245$
PYL $1325 \mathrm{~W} / 1-259$ PFL11 $0203 / 1-263$ PF14-0616/1-252 PF11-0175/1-235 PY11 $0175 / 1.236$ PP14- $0063 / 1-245$ raLe $1.92 / 1-255$ PrCO140e/1-256 PP14 $0548 / 1=245$
PFEOI $55 \mathrm{w} / 1-274$

N-teminal helix Strand:

Helix!

WalkerB

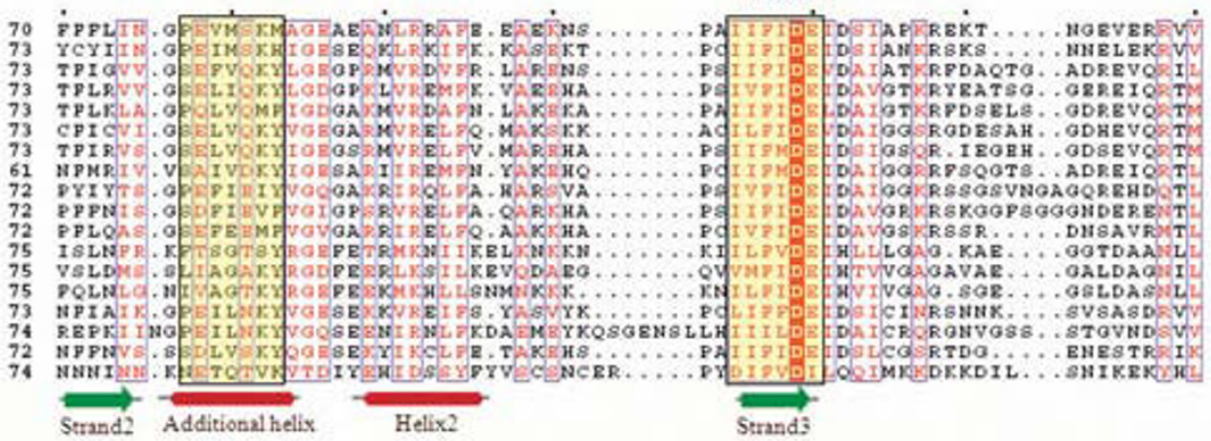

Strand Additionalhelix Helix

Singing

Sensorl Arginine finger

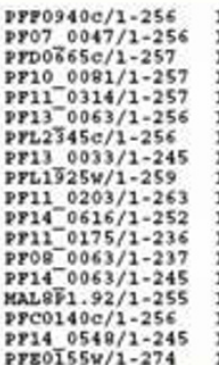

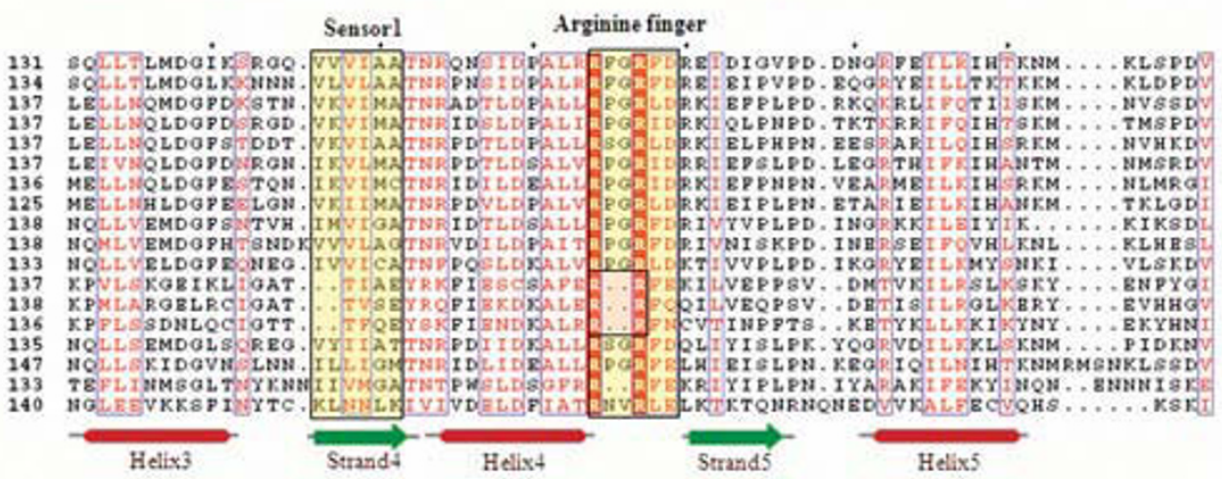

DPF0940c/1-256 PF07 0047/2-256 PrD0665e/1-257 PF10_0081/1-25? $P P 11-0314 / 1-257$
$P P 13-0053 / 1-256$ PL.2345c/1-256 $P F 130033 / 1-245$ PF11 0203/1-263 PP14-0616/1-252 pP11-0175/1-236 $P P 0 Q^{-} 0063 / 1-237$ PF14-0063/1-245 MaL $851.92 / 1=255$ P $9 \mathrm{CO} 140 \mathrm{c} / 1-256$ PY14 0548/1-245
X.LELRSMTK.
Sensor2
ULRKI AKRCK
ipVord
DLBEPVMBED.
axvand
ELSOADIKAICTEAGLLALPERRME. ITTADLRKARDKALFQK.
RPRLL
DMLKIATDMN . .... NC GA BVKAVCTEAGMPALRERRVH, VTQEDPBMAVAKVMKQD
DYBSVCRLCD ....GPIG DLRNVCTEAGMPATRAMRDY, VIBEDFYKAARKI \&EOK.
KLBDIEKIAR. LTPGP GADLENVVNEATILATRMBKBL, VTINEL TEARDKVBMGP
DIKUI YILASLTPOPVO DIAUVVNEOAIQCARRSMLLOVQI KDPELAIERVI
DLRVL RRTV.... GMTQ DLMILNIAAIRCSVRCK
NITDKALVAA. A. KI D PIKDKXLPDKAIDLLN.
YYTDD LVA...
MYRI KLTR...... OY OADLYOVLRESAPIALOECRD
MILELARRTP.....MPSOARIRLVRHTVSYAPERHI
DIKQPATLTE.......
ILIGI $2 N B L D$
Helix6
Helix 7

\section{Figure 7}

Multiple sequence alignment of P-loop NTPase domain of $P$. falciparum classical AAA and ClpA/B proteins.

Conserved residues are in solid red and characteristic motifs are boxed in black and shaded in yellow. The corresponding secondary structure is shown below the alignment. Red cylinder represents helices and green arrows represent $\beta$-strands. Note the presence of additional helix between strand 2 and helix 2 in these proteins. Also note the lack of conserved glycine in Clp proteins, upstream of arginine finger (boxed in arginine finger). 


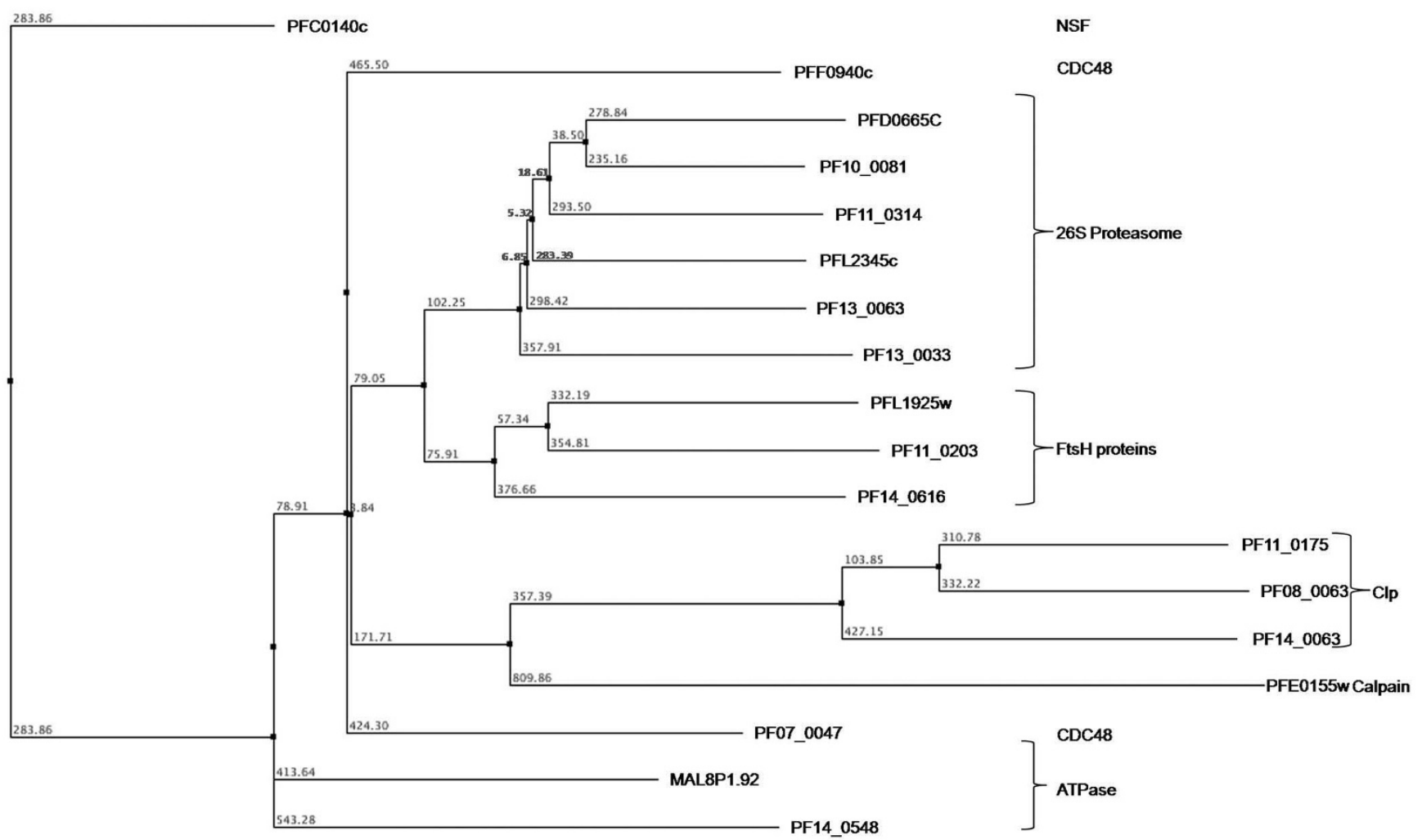

Figure 8

Phylogenetic classification of $P$. falciparum AAA and CIpA/B proteins based upon sequence and secondary structure homology among their P-loop domain. The branch numbers represents the distance values in $\mathrm{NJ}$ tree of protein sequences.

chain domains at their N-terminal named as DHC N-1 and DHC N-2 except in MAL7P1.89 and PF11_0240, which only have the DHC N-2 domain. The PFI0260C protein is also found to contain spectrin-like repeats.

\section{AAA clade}

Proteins belonging to the classical AAA clade contain highly conserved P-loop NTPase domain including SRH. The characteristic feature of the clade is the presence of an additional short helix immediately downstream of strand 2 in the P-loop domain [35]. The AAA clade consists of all ATPases that originally are defined as the members of the AAA superfamily [44]. The $P$. falciparum AAA proteins have been classified under following categories: metalloproteases, proteasomal subunits and 'D1 and D2' proteins. A conserved glycine residue at the $\mathrm{N}$-terminal of the arginine finger is observed in all the members of this clade (Figure 7). Three P. falciparum proteins are identified as metalloproteases (pan-bacterial protein family) belonging to the M41 family of peptidases and proteases $[45,46]$. PFL1925w has been annotated as cell-division protein FtsH whereas PF11_0203 and PF14_0616 have been annotated as hypothetical proteins (PlasmoDB). The domain analysis of these proteins showed that these hypothetical proteins contain a single AAA (Pfam domain id-PF00004) domain fused to the metalloprotease domain (Pfam domain id-PF01434) as in case of other FtsH proteins. Moreover, in all the three metalloproteases, a functional motif 'abXHEbbHbc' where 'a' is most often alanine or serine (instead of valine or threonine residues observed in metalloproteases of other organisms), ' $b$ ' is an uncharged residue (tyrosine or alanine here) and ' $c$ ' is a hydrophobic residue (leucine or isoleucine here) is observed. Thus both, domain and sequence-structure analysis, suggested that the two hypothetical proteins with characteristic features of the family may be functionally equivalent to FtsH metalloprotease. Upon performing the phylogenetic analysis based on their secondary structures, these three proteins tend to cluster together.

The 26S proteasomes complex is a component of the regulated protein degradation machinery in eukaryotic cell. The 19S regulatory component of the $26 \mathrm{~S}$ proteasome complex consists of six distinct but closely related proteins 

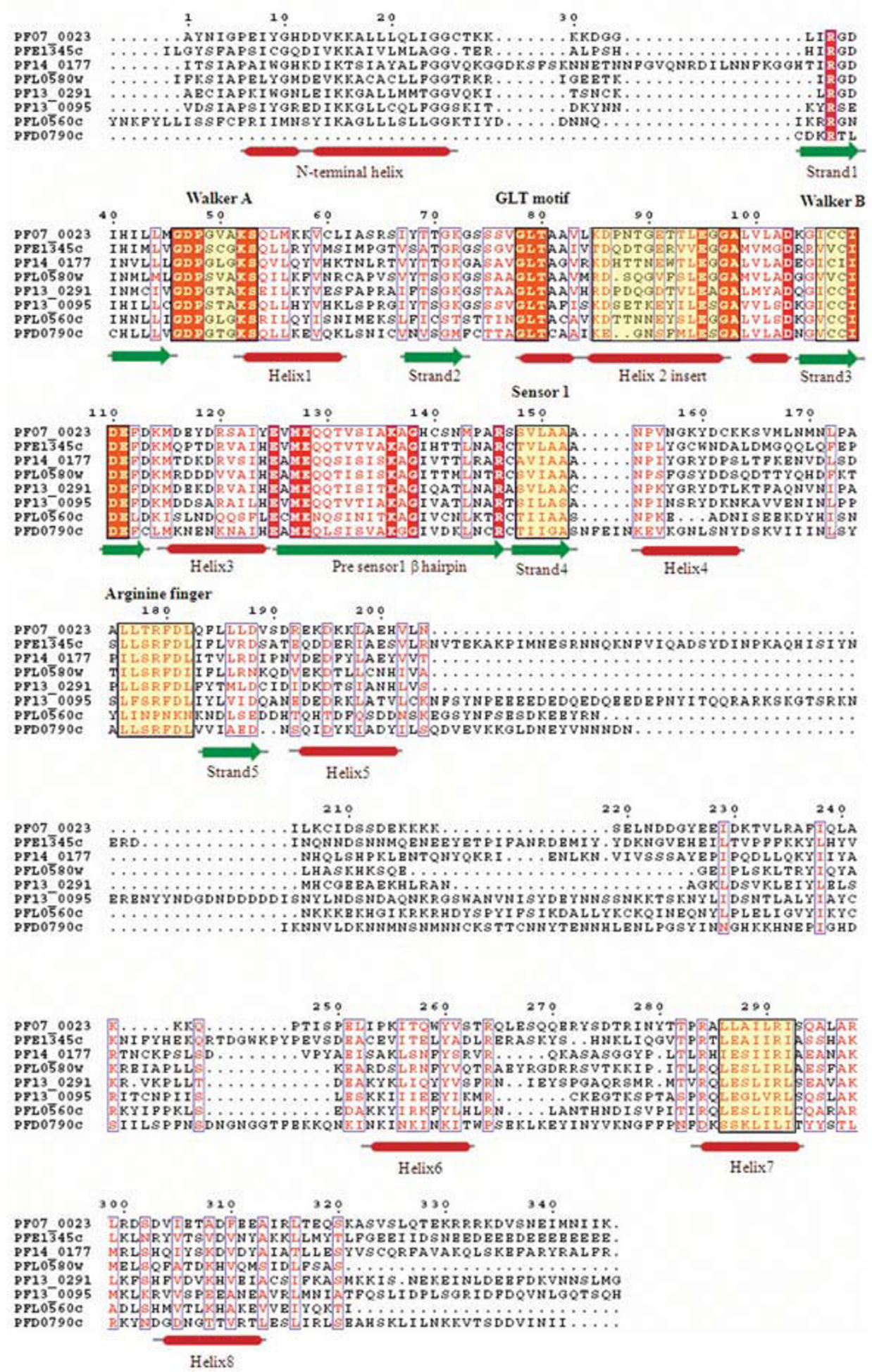

Figure 9

Multiple sequence alignment of $P$-loop NTPase domain of $\boldsymbol{P}$. falciparum Mini chromosomal maintenance

(MCM) proteins. Conserved residues are in solid red and characteristic motifs are boxed in black and shaded in yellow. The corresponding secondary structure is shown below the alignment. Red cylinder represents helices and green arrows represent $\beta$-strands. Note the presence of helix 2 insert and Pre-sensor I hairpin characteristic of this family. 
(Rpt1-6) [47,48]. These proteasomal ATPases contain tandem repeat of AAA module and are conserved throughout the archeao-eukaryotic branch $[49,50]$. In the P. falciparum genome database, five proteins (PFD0665c PF10_0081, PF11_0314, PF13_0033 and PF13_0063) have already been identified as Rpt homologues. Another protein PFL2345c, which is annotated as the tat-binding protein homolog, has also been found to be the part of this complex based on its sequence similarity and structural patterns. Secondary structure analysis shows that all six the proteins have classical helical and $\beta$-strand pattern (Figure 7). The 'D1 and D2' is another family that contains proteins with two AAA domains named as D1 and D2. This family includes $\mathrm{N}$-ethylmaleimide-sensitive fusion protein (NSF), ATPase family gene (AFG) and Cell Division Cycle 48 (CDC48) proteins [51,52]. The NSF proteins play an important role in vesicle mediated protein trafficking in which the D1 AAA cassette is the active ATPase while D2 is nucleotide binding $[47,48]$. The only NSF homologue identified in the malaria parasite is PFC0140c that contains the N-domain (essential for soluble NSF-attachment protein binding). The ATPase CDC48 family has two representatives in the $P$. falciparum genome, PF07_0047 and PFF0940c. An additional protein (MAL8P1.92) is observed, having similar sequence features and structural as that of known CDC48 proteins. It shows a significant sequence similarity of MAL8P1.92 with known CDC48 proteins along with the conserved secondary structural features such as the presence of an additional helix downstream of strand-2 in the ATPase domain. Phylogenetic analysis clustered proteins of proteasomal complex in a tight cluster close to another cluster of FtsH proteins (Figure 8).

\section{RuvB/TIP49 clade}

The TIP49 ATPases associate with the TATA-binding proteins and appear to play a critical role in the assembly of complexes related to transcriptional activation [53]. Three proteins (PF11_0071, PF08_0100 and PF13_0330) have already been annotated as RuvB DNA helicases in the $P$. falciparum genome. However, presence of certain key features such as conserved 'pre-sensor- $1 \beta$ hairpin' which is the characteristic of RuvB DNA helicases is not observed in these three proteins. Instead, the N-terminal module with $\mathrm{H}$ [ST]H motif, a characteristic of TIP49 family $[35,54]$ is found in these proteins. Furthermore, the multiple sequence alignment based secondary structure analysis showed presence of an additional small helix downstream of strand 2, as in the case of classical AAA clade proteins (Figure 10). These observations suggest that these three proteins should be annotated as TIP49 proteins in the classical AAA clade instead of RuvB DNA helicases.

\section{MutS proteins/DNA mismatch repair proteins}

The precise cladistic classification of this group of proteins is not known due to the lack of any unique/peculiar feature(s) pertaining to this group of proteins. In the P. falciparum genome database, three proteins (MAL7P1.206, PF14_0254 and PFE0270c) have been annotated as DNA mismatch repair proteins. The characteristic motifs (Walker A, STF, Walker B and STH) for this class of proteins are found to be well conserved in all the three proteins. A hypothetical protein PF14_0051 is also found to contain similar domain as other MutS proteins but showed sequence variations all along the length of P-loop domain, including considerable substitution of ' $\mathrm{T}$ ' $\rightarrow$ ' $\mathrm{L}$ ' in the STF motif (Figure 11). Phylogenetic classification based on the secondary structure of the P-loop domains of these proteins grouped them within the cluster of five $\mathrm{ABC}$ transporter proteins (Figure 12) and thus together these can be classified under the ABC/SMC superfamily.

\section{AAA domain sequence and secondary structure based functional classification of hypothetical proteins}

The phylogenetic analyses of all the 97 P. falciparum Ploop NTPases based upon the secondary structure as well as sequence features of their AAA domain led to an interesting observation. The functional distribution of all 97 proteins is shown as a percentage of total number of proteins for each of the clade studied (Figure 13). Proteins with similar functional roles (or which are parts of larger functional complexes) are found to be grouped together in the analysis. This is remarkably observed for $\mathrm{FtsH}$, ClpA/B and dynein ATPases families. A number of hypothetical proteins with characteristic functional domains are also found to strongly cluster with the annotated proteins of respective families. For example, the two hypothetical proteins having FtsH domains (PF11_0203 and PF14_0616) clustered with the annotated FtsH protein when subjected to both sequence and secondary structure based analyses (Figure 12). A similar result is found for the hypothetical protein PFI0260c, having dynein heavy chain domain, which clusters with four other dynein proteins (Figure 12). These results suggest a correlation between the sequence and structure conservation of the Ploop domain and the functional role of these proteins. Thus it can be speculated that proteins involved in similar functions may group together due to conservation of structural and sequence patterns of their NTPase domains and thus may be suggestive of their putative role. A number of hypothetical proteins that contain only AAA domain are also found to group within clusters of proteins having a defined functional role (Figure 12). These results suggest that the AAA domain sequence and secondary structure homology based phylogenetic grouping may help to infer possible functional role of these AAA pro- 


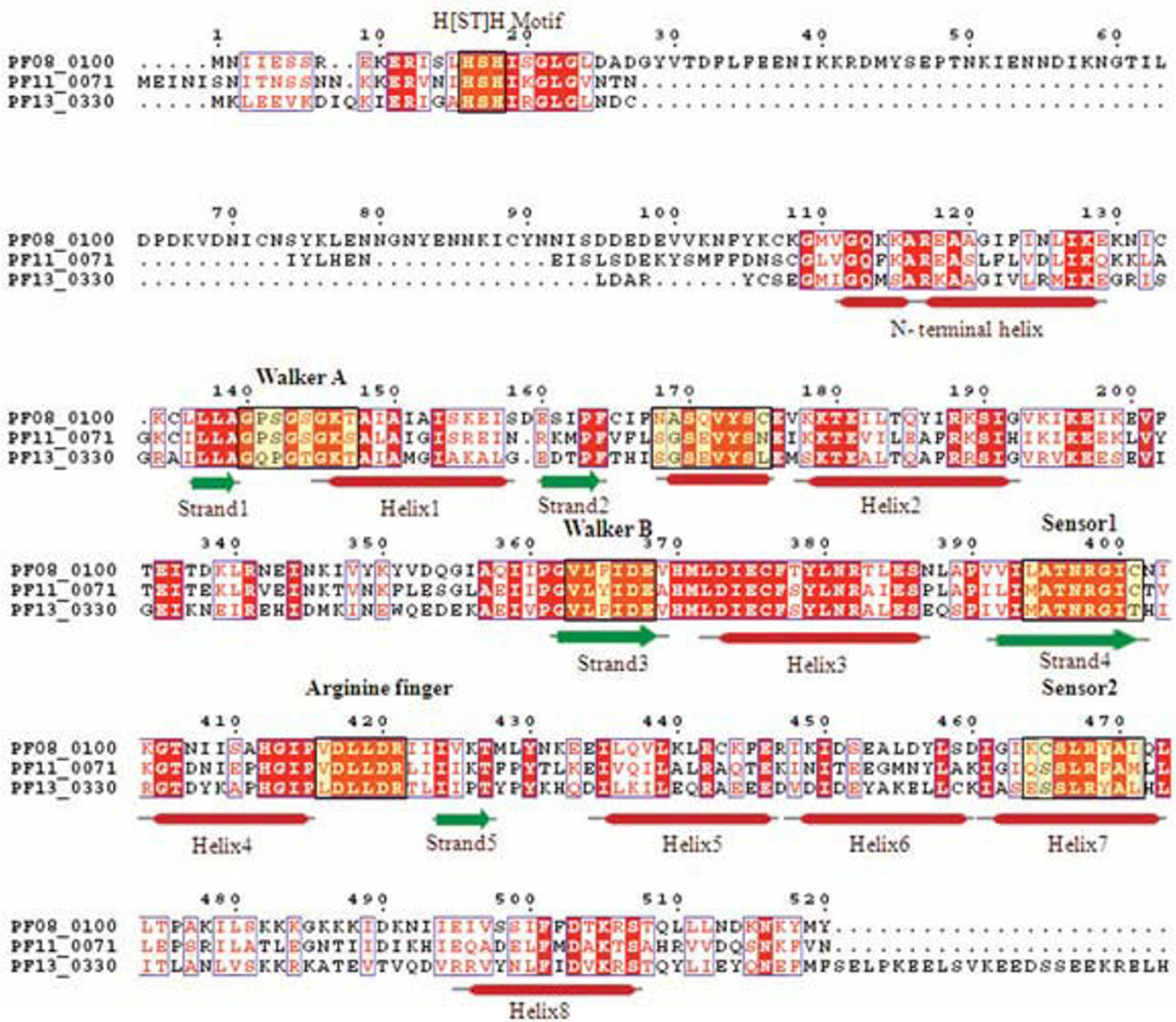

\section{Figure 10}

Multiple sequence alignment of P-loop NTPase domain of $P$. falciparum Tip 49 proteins. Conserved residues are in solid red and characteristic motifs are boxed in black and shaded in yellow. The corresponding secondary structure is shown below the alignment. Red cylinder represents helices and green arrows represent $\beta$-strands. Note the presence of additional helix between strand 2 and helix 2 in these proteins.

teins and may be used as a complementary method in the annotation process.

Absence of sequence similarity with any other known proteins is a major hurdle in functional classification of large number of $P$. falciparum hypothetical proteins. Martin [55] used hydropathy plots to identify novel membrane transporters from these hypothetical proteins of $P$. falciparum. The possible functional roles for few of $P$. falciparum hypothetical proteins are predicted based upon their grouping with other functionally annotated P-loop proteins in the phylogenetic analysis. The PF14_0126 protein is found to cluster with two functionally annotated RFC proteins and PFD0935c is observed to fall within the cluster of three MCM proteins and thus these proteins might have functional roles as RFC and MCM proteins respectively. Similarly PFF0810c and PF10_0099 are observed to cluster with SMC proteins, MAL13P1.13 with $\mathrm{ABC}$ transporters and PF14_0052 with ran/tc4 family proteins, suggesting their possible functional roles as $\mathrm{SMC}$, $\mathrm{ABC}$ transporters and ran/tc4 proteins respectively. These hypothetical proteins have shown significant similarities 


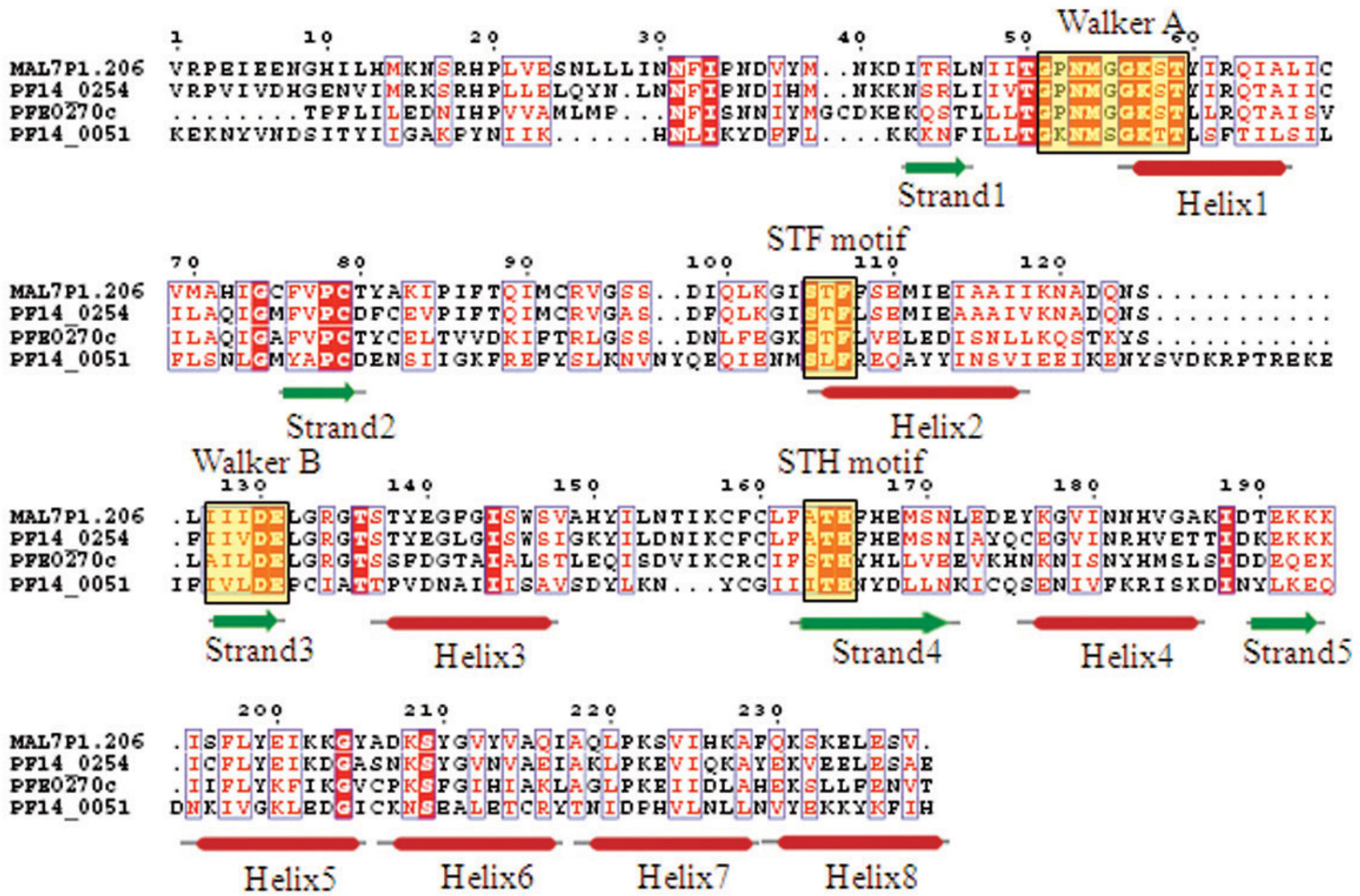

Figure I I

Multiple sequence alignment of $\mathbf{P}$-loop NTPase domain of $\boldsymbol{P}$. falciparum MutS proteins. Conserved residues are in solid red and characteristic motifs are boxed in black and shaded in yellow. The corresponding secondary structure is shown below the alignment. Red cylinder represents helices and green arrows represent $\beta$-strands.

at both the sequence and secondary structural levels. Thus, this approach may be extended to other organisms to classify and assign putative functions to many hypothetical proteins.

\section{Identification of orthologous protein sequences using OrthoMCL}

Firstly, 1,580 different orthologous groups are found between $P$. falciparum and Homo sapiens, consisting of 1,683 different proteins from the parasite. Interestingly, 37 P. falciparum P-loop NTPases have no ortholog in $H$. sapiens. These proteins include helicases, $\mathrm{ABC}$ transporters, Clp protease and heat shock protein. Out of these 37 proteins, 17 are annotated as hypothetical proteins. Together, this analysis offers opportunities to explore the potential of these proteins as novel drug targets without affecting the host.

The OrthoMCL analysis amongst six Plasmodium sp. namely $P$. falciparum, $P$. vivax, $P$. berghei, $P$. chabaudi, $P$. yoelii and $P$. knowlesi, identified 88 different $P$. falciparum
P-loop NTPase proteins that have at least one ortholog in another malaria parasite species. The remaining nine NTPase proteins of $P$. falciparum are the proteins, which are unique to $P$. falciparum genome. These proteins include $\mathrm{ABC}$ transporter, 26S proteasome subunit Rpt3 and DNA replication licensing factor MCM2 in addition to hypothetical proteins. These proteins might be responsible for some dedicated pathways in the life-cycle of the parasite and thus can be of immense interest for further research that may provide clues to a number of unanswered questions in the parasite biology.

Since, there are few characteristic similarities among $P$. falciparum and plants; an attempt is made to identify orthologous of $P$. falciparum P-loop NTPase proteins in Arabidopsis thaliana a model organism to compare. A total of 57 parasite NTPase proteins have ortholog in A. thaliana genome. Four of these proteins are predicted to be targeted to the apicoplast of the P. falciparum. These are cell division cycle ATPase (PF07_0047), heat shock protein (PF11_0175), ATP-dependent transporter 


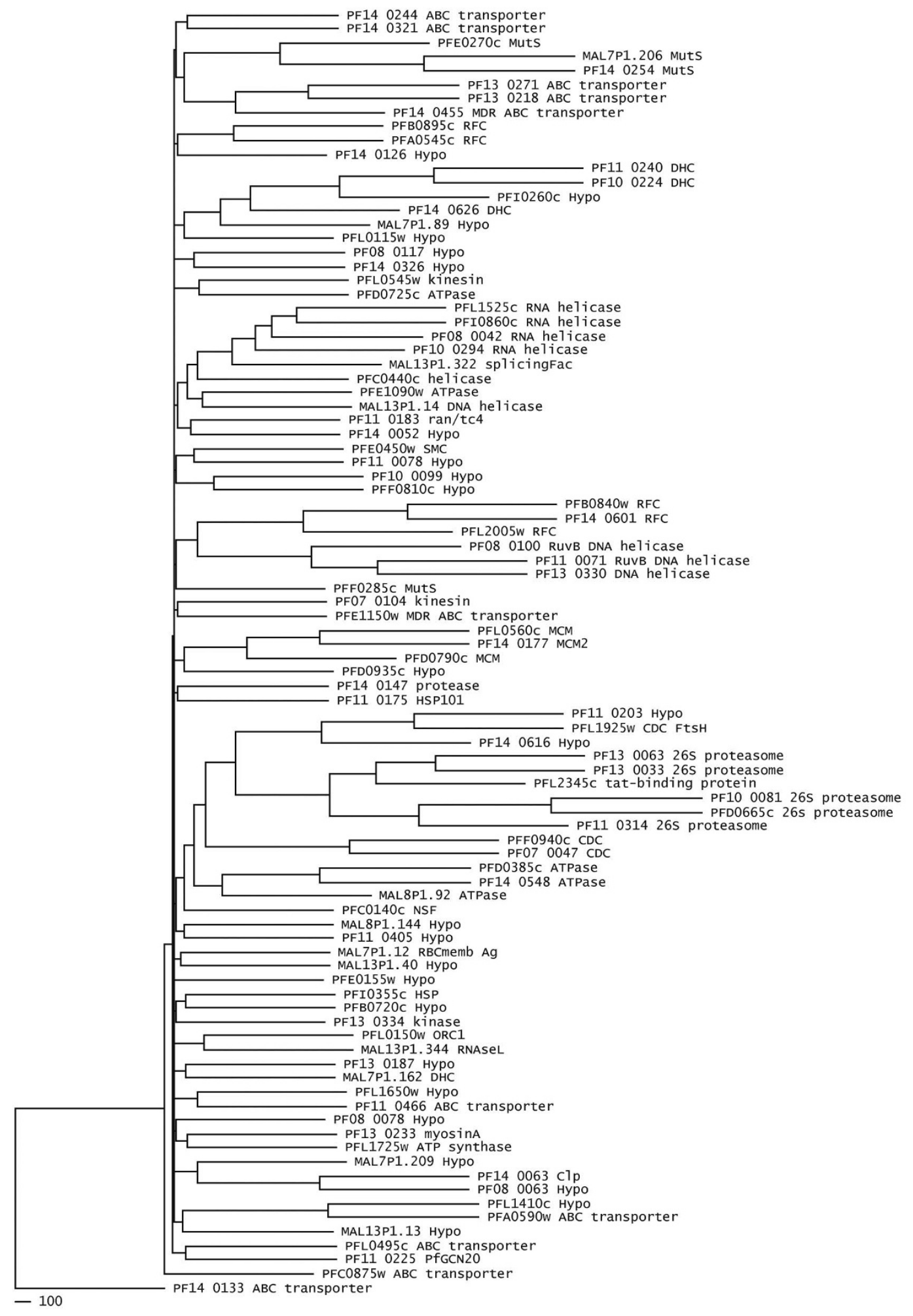

Figure 12

The dendrogram showing phylogenetic grouping based on the sequence and structural similarity of NTPase domain of $\boldsymbol{P}$. falciparum $\mathbf{P}$-loop NTPases. The proteins with their respective annotations are mentioned. 


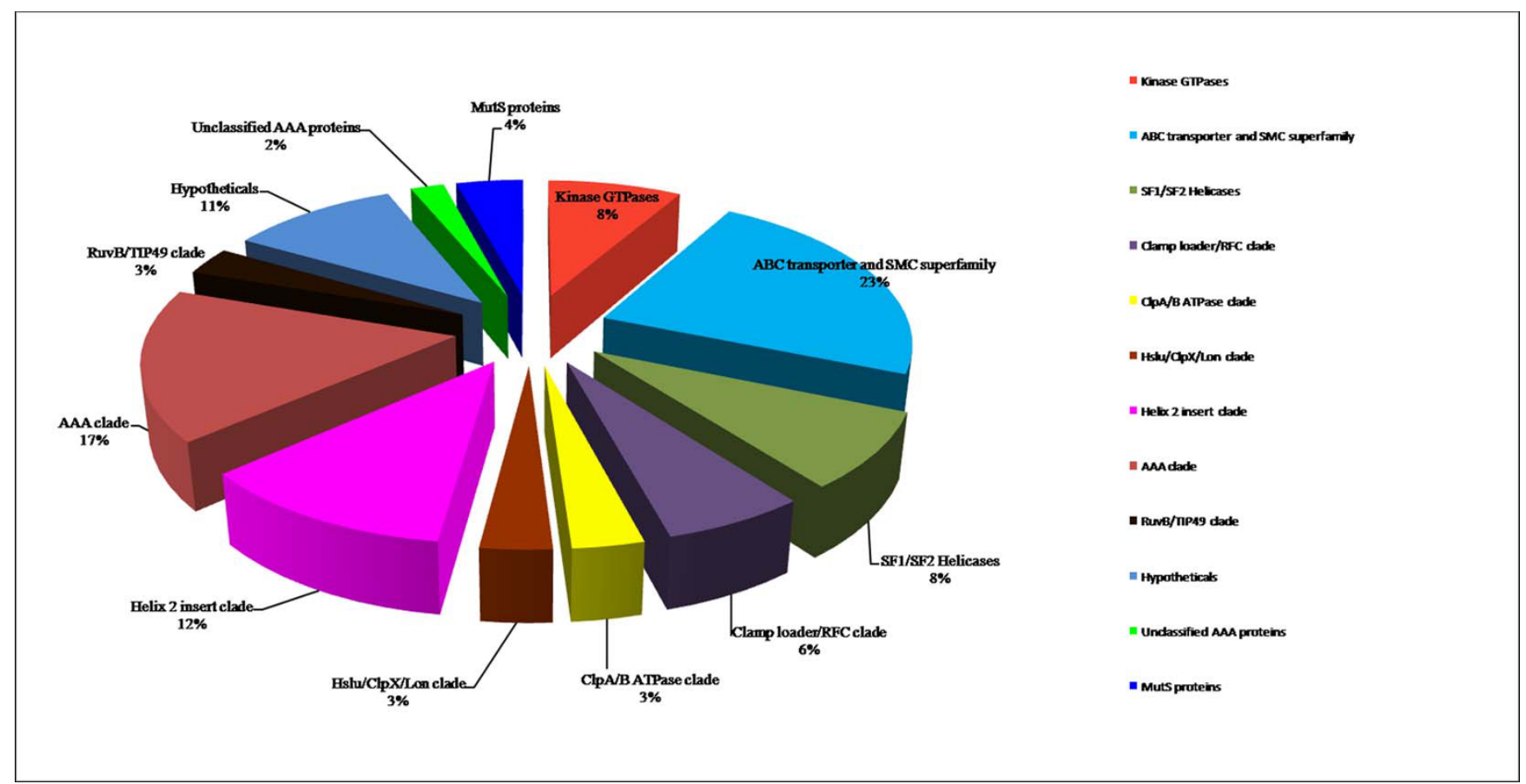

Figure 13

Functional distribution of P-loop NTPases in the studied dataset.

(PF14_0133) and a hypothetical protein (PF08_0063). Out of these four proteins, the ATP-dependent transporter protein has no human ortholog. The expression profile of this protein shows the peak expression at late-trophozoite and early schizont stages of the life-cycle. Together, this protein may be considered as a promising drug target.

The orthologous search is further extended to prokaryotes (Synechococcus sp., Mycobacterium tuberculosis, Escherichia coli and Staphylococcus aureus) where only 10 P. falciparum P-loop NTPases are observed to have orthologs in at least one of these prokaryotes. These 10 NTPases from the parasite showed sequence or structural similarity with bacterial ATPases such as multidrug resistance protein; heat shock protein and cell division protein FtsH protein (present in all eubacterial species). It strongly suggests that few unique processes of the parasite are governed by prokaryotic type mechanisms such as drug-resistance, cellcycle or protein folding involving heat shock proteins. Some of these prokaryote like-parasite proteins may play crucial role in the parasite life cycle and can be studied as novel drug targets.

\section{Concluding remarks}

P-loop NTPases comprise one of the largest protein families with members present in all kingdoms of life. Numerous subgroups of the family are involved in diverse cellular functions. The functional roles of a number of families belonging to P-loop NTPases superfamily are still unknown in eukaryotes. The repertoire of the P-loop NTPase has not been identified and classified in $P$. falciparum. The challenge of studying and classifying these NTPases further increases in $P$. falciparum due to low sequence similarity and unique features of the genome such as extended insertions and repeats. In the present study, a systematic classification of the P-loop NTPases in $P$. falciparum genome is carried out, that provided information on their function, classification, phylogenetic and orthologous relationships amongst various protein families and organisms. Variations in critical residues within the conserved regions as well as long insertions are observed in the P-loop NTPase domain for most of the $P$. falciparum NTPases suggesting that the parasite has evolved constantly to sustain inspite of the mutations/variations in these imperative regions. The study provided an understanding of the P-loop NTPases, especially in terms of their structural and functional relationships. The proteins with similar functional roles are observed to have similar sequence and structure pattern of P-loop domain. Based on this, putative functional roles for 14 hypothetical proteins are predicted. This is one of the key findings of the study pertaining to the fact that most of $P$. falciparum proteins are not homologous to any other eukaryotic protein and have been annotated as hypothetical proteins. Therefore, elucidation of putative roles of these proteins that are unique to the parasite may provide leads 
to identify novel drug targets. The sequence orthology based studies are found to be useful in identifying P-loop NTPases either similar to prokaryotic origin or restricted to Plasmodium species. Such P-loop NTPases involved in important physiological pathways may lead to identification of new drug targets. It must be emphasized that the current study demonstrates the possible achievements of a computational analysis and is a preliminary investigation. Experimental evidence to explore the role of these genes is thus required. It becomes mandatory in the case of $P$. falciparum where new functional roles have been predicted for a significant number of hypothetical proteins inspite of very low levels of sequence similarity. Overall the study provides us new leads in investigating the functions and biology of $P$. falciparum P-loop NTPases.

\section{Competing interests}

The authors declare that they have no competing interests.

\section{Authors' contributions}

$\mathrm{DG}^{1}$ and MKK carried out the work and drafted the manuscript. DG ${ }^{2}$ and VSC critically revised the manuscript content. AM contributed to concept, design and have given final approval of the version to be published.

\section{Additional material}

\section{Additional file 1}

A brief description of different clades of P-loop NTPases showing number of proteins in each class, respective protein ID, full sequence length and the P-loop NTPase region under study. Additional table. Click here for file

[http://www.biomedcentral.com/content/supplementary/14752875-8-69-S1.doc]

\section{Additional file 2}

Table showing repertoire of P-loop NTPases of P. falciparum and their sequence characteristics. Expression profiles of each protein across the parasitic life cycle based on the transcriptome data as well as their annotation at PlasmoDB are also given. The hypothetical proteins which are assigned functional roles in this study are boxed and highlighted in bold. Proteins orthologous to prokaryotes are marked with \pm and those orthologous to Arabidopsis thaliana are marked with *. SS, Signal Sequence; TM, trans-membrane region; $S$, sporozoite; ER, early ring; $L R$, late ring; ET, early trophozoite; $E S$, early schizont; $L S$, late schizont; and $M$, merozoites.

Click here for file

[http://www.biomedcentral.com/content/supplementary/14752875-8-69-S2.doc]

\section{Additional file 3}

Evolutionary classification of $\mathrm{P}$. falciparum $\mathrm{ClpA} / \mathrm{B}$ proteins with seed ClpA/B proteins extracted from Pfam (Pfam domain idPF10431). Additional figure.

Click here for file

[http://www.biomedcentral.com/content/supplementary/14752875-8-69-S3.tiff]

\section{Acknowledgements}

DG and MKK are supported by research fellowship from Council of Scientific and Industrial Research, India. The research in lab of AM is supported by Department of Biotechnology, Govt. of India.

\section{References}

I. Snow RW, Guerra CA, Noor AM, Myint HY, Hay SI: The global distribution of clinical episodes of Plasmodium falciparum malaria. Nature 2005, 434:2I4-2I7.

2. McConkey AG, Pinney WJ, Westhead RD, Plueckhahn K, Fitzpatrick BT, Macheroux P, Kappes B: Annotating the Plasmodium genome and the enigma of the shikimate pathway. Trends Parasitol 2004, 20:60-65.

3. Koonin EV, Aravind L: The NACHT family - a new group of predicted NTPases implicated in apoptosis and MHC transcription activation. Trends Biochem Sci 2000, 25:223-224.

4. Saraste M, Sibbald PR, Wittinghofer A: The P-loop - a common motif in ATP- and GTP-binding proteins. Trends Biochem Sci 1990, 15:430-434.

5. Vetter IR, Wittinghofer A: Nucleoside triphosphate-binding proteins: different scaffolds to achieve phosphoryl transfer. Q Rev Biophys 1999, 32:1-56.

6. Mogk A, Dougan D, Weibezahn J, Schlieker C, Turgay K, Bukau B: Broad yet high substrate specificity: the challenge of AAA+ proteins. I Struct Biol 2003, 146:90-98.

7. Walker JE, Saraste M, Runswick MJ, Gay NJ: Distantly related sequences in the alpha- and beta-subunits of ATP synthase, myosin, kinases and other ATP requiring enzymes and a common nucleotide binding fold. EMBO J 1982, I:945-95 I.

8. Milner-White EJ, Coggins JR, Anton IA: Evidence for an ancestral core structure in nucleotide-binding proteins with the type A motif. J Mol Biol 1991, 22 1:751-754.

9. Neuwald AF, Aravind L, Spouge JL, Koonin EV: AAA+: A class of chaperone-like ATPases associated with the assembly, operation, and disassembly of protein complexes. Genome Res 1999, 9:27-43.

10. Fröhlich KU: An AAA family tree. J Cell Sci 200I, I I4:160I-I602.

II. Frickey T, Lupas AN: Phylogenetic analysis of AAA proteins. J Struct Biol 2004, 146:2-10.

12. Lupas AN, Martin J: AAA proteins. Curr Opin Struct Biol 2002, I 2:746-753.

13. Altschul SF, Madden TL, Schäffer AA, Zhang J, Zhang Z, Miller W, Lipman DJ: Gapped BLAST and PSI-BLAST: a new generation of protein database search programs. Nucleic Acids Res 1997, 25:3389-3402.

14. Thompson JD, Gibson TJ, Plewniak F, Jeanmougin F, Higgins DG: The CLUSTAL $X$ windows interface: flexible strategies for multiple sequence alignment aided by quality analysis tools. Nucleic Acids Res 1997, 25:4876-4882.

15. Notredame C, Higgins DG, Heringa J: T-Coffee: a novel method for fast and accurate multiple sequence alignment. J Mol Biol 2000, 302:205-217

16. Wolf YI, Rogozin IB, Kondrashov AS, Koonin EV: Genome alignment, evolution of prokaryotic genome organization, and prediction of gene function using genomic context. Genome Res 200I, I I:356-372.

17. Felsenstein J: PHYLIP Phylogeny Inference Package 3.5. Department of Genetics, The University of Washington, Seattle, WA; 1993

18. Dayhoff M: Atlas of Protein Sequence and Structure. National Biomedical Research Foundation, Washington, DC; 1978.

19. Li L, Stoeckert CJ Jr, Roos DS: OrthoMCL: Identification of ortholog groups for eukaryotic genomes. Genome Res 2003, 13:2178-89.

20. Leipe DD, Wolf YI, Koonin EV, Aravind L: Classification and evolution of P-loop GTPases and related ATPases. J Mol Biol 2002, 317:41-72

21. Leipe DD, Koonin EV, Aravind L: Evolution and classification of P-loop kinases and related proteins. I $\mathrm{Mol}$ Biol 2003, 333:78I-8I5

22. Anamika , Srinivasan N, Krupa A: A Genomic Perspective of Protein Kinases in Plasmodium falciparum. Proteins: Structure, Function, and Bioinformatics 2005, 58:180-189. 
23. Dean M, Rzhetsky A, Allikmets R: The Human ATP-Binding Cassette (ABC) Transporter Superfamily. Genome Research 200I I I:I I56-I I66.

24. Klokouzas A, Barrand MA, Hladky SB: Effects of clotrimazole on transport mediated by multidrug resistance associated protein I (MRPI) in human erythrocytes and tumour cells. Eur J Biochem 200I, 268:6569-6577.

25. Bozdech Z, VanWye J, Haldar K, Schurr E: The human malaria parasite Plasmodium falciparum exports the ATP-binding cassette protein PFGCN20 to membrane structures in the host red blood cell. Mol Biochem Parasitol 1998, 97:81-95.

26. Bozdech Z, Llinas M, Pulliam BL, Wong ED, Zhu J, DeRisi JL: The Transcriptome of the Intraerythrocytic Developmental Cycle of Plasmodium falciparum . PLOS Biol 2003:E5.

27. Ginsburg $\mathrm{H}$ : Progress in in-silico functional genomics: the malaria Metabolic Pathways database. Trends Parasitol 2006, 22:238-40

28. Bodó A, Bakos E, Szeri F, Váradi A, Sarkadi B: The role of multidrug transporters in drug availability, metabolism and toxicity. Toxicol Lett 2003, |40-141:133-|43.

29. Lohman TM, Bjomson KP: Mechanisms of helicase-catalyzed DNA unwinding. Annu Rev Biochem 1996, 65:169-214.

30. Gorbalenya AE, Koonin EV: An NTP-binding motif is the most conserved sequence in a highly diverged monophyletic group of proteins involved in positive strand RNA viral replication. J Mol Evol 2003, 28:256-268.

31. Koonin EV, Dolja VV: Evolution and taxonomy of positivestrand RNA viruses: implications of comparative analysis of amino acid sequences. Crit Rev Biochem Mol Biol I 993, 28:375-430.

32. Singleton MR, Wigley $D B$ : Modularity and specialization in superfamily I and 2 helicases. I Bacteriol 2002, I 84: |8|9-|826.

33. Vale RD: AAA proteins. Lords of the ring. I Cell Biol 2000, I 50:FI3-9.

34. Hanson IP, Whiteheart SW: AAA+ proteins: have engine, will work. Nat Rev Mol Cell Biol 2005, 6:5 19-29.

35. lyer LM, Leipe DD, Koonin EV, Aravind L: Evolutionary history and higher order classification of AAA+ ATPases. J Struct Biol 2004, |46:||-3|.

36. Hoskins JR, Sharma S, Sathyanarayana BK, Wickner S: Clp ATPases and their role in protein unfolding and degradation. Adv Protein Chem 2001, 59:4I3-429.

37. Kwon AR, Kessler BM, Overkleeft HS, McKay DB: Structure and reactivity of an asymmetric complex between HsIV and Idomain deleted HsIU, a prokaryotic homolog of the eukaryotic proteasome. I Mol Biol 2003, 330:185-195.

38. Song HK, Bochtler M, Azim MK, Hartmann C, Huber R, Ramachandran R: Isolation and characterization of the prokaryotic proteasome homolog HsIVU (ClpQY) from Thermotoga maritima and the crystal structure of HsIV. Biophys Chem 2003 , 100:437-52

39. Couvreur B, Wattiez R, Bollen A, Falmagne P, Le Ray D, Dujardin JC: Eubacterial HsIV and HsIU subunits homologs in primordial eukaryotes. Mol Biol Evol 2002, 19:21 I0-2। I7.

40. Bochtler M, Hartmann C, Song HK, Bourenkov GP, Bartunik HD, Huber R: The structures of HsIU and the ATPdependent protease HsIU-HsIV. Nature 2000, 403:800-805.

4I. Ramasamy G, Gupta D, Mohmmed A, Chauhan VS: Characterization and localization of Plasmodium falciparum homolog of prokaryotic ClpQ/HsIV protease. Mol Biochem Parasitol 2007, 152:139-48.

42. Koonin EV: A common set of conserved motifs in a vast variety of putative nucleic acid-dependent ATPases including MCM proteins involved in the initiation of eukaryotic DNA replication. Nucleic Acids Res 1993, 2 1:254 I-2547.

43. Mocz G, Gibbons IR: Model for the motor component of dynein heavy chain based on homology to the AAA family of oligomeric ATPases. Structure 2001, 9:93-103.

44. Confalonieri F, Duguet M: A 200-amino acid ATPase module in search of a basic function. Bioessays 1995, 17:639-650.

45. Rawlings ND, Barrett AJ: Evolutionary families of metallopeptidases. Methods Enzymol 1995, 248:183-228.

46. Rawlings ND, Barrett AJ: MEROPS: The peptidase database. Nucleic Acids Res 1999, 27:325-31.

47. Ogura T, Wilkinson AJ: AAA+ superfamily ATPases: common structure-diverse function. Genes Cells 200 I, 6:575-97.
48. Ogura T, Whiteheart SW, Wilkinson AJ: Conserved arginine residues implicated in ATP hydrolysis, nucleotidesensing, and inter-subunit interactions in AAA and AAA+ ATPases. Struct Biol 2004, I 46: 106-I I 2

49. Beyer A: Sequence analysis of the AAA protein family. Protein Sci 1997, 6:2043-2058.

50. Swaffield JC, Purugganan MD: The evolution of the conserved ATPase domain (CAD): reconstructing the history of an ancient protein module. J Mol Evol 1997, 45:549-63.

5I. Lee YJ, Wickner RB: AFG I, a new member of the SEC I 8- NSF, PASI, CDC48-VCP, TBP family of ATPases. Yeast 1992, 8:787-90

52. Ye Y, Meyer HH, Rapoport TA: The AAA ATPase Cdc48/p97 and its partners transport proteins from the $E R$ into the cytosol. Nature 200I, 4I 4:652-656.

53. Wood MA, McMahon SB, Cole MD: An ATPase/helicase complex is an essential cofactor for oncogenic transformation by c-Myc. Mol Cell 2000, 5:321-330.

54. Kurokawa Y, Kanemaki M, Makino Y, Tamura TA: A notable example of an evolutionary conserved gene: studies on a putative DNA helicase TIP49. DNA Seq 1999, 10:37-42.

55. Martin RE, Henry RI, Abbey JL, Clements JD, Kirk K: The 'permeome' of the malaria parasite: an overview of the membrane transport proteins of Plasmodium falciparum . Genome Biol 2005, 6:R26.

Publish with Bio Med Central and every scientist can read your work free of charge

"BioMed Central will be the most significant development for disseminating the results of biomedical research in our lifetime. "

Sir Paul Nurse, Cancer Research UK

Your research papers will be:

- available free of charge to the entire biomedical community

- peer reviewed and published immediately upon acceptance

- cited in PubMed and archived on PubMed Central

- yours - you keep the copyright 